\title{
Effectiveness of the WHO-Authorized COVID-19 Vaccines: A Rapid Review of Global Reports till 30 June 2021
}

\author{
Chang-Jie Cheng ${ }^{1,2}$, Chun-Yi Lu ${ }^{3,4}$ (D), Ya-Hui Chang ${ }^{5}$, Yu Sun ${ }^{1,2}$,, Hai-Jui Chu ${ }^{1,2}$, Chun-Yu Lee ${ }^{1,2}$, \\ Chang-Hsiu Liu ${ }^{1}$, Cheng-Huai Lin ${ }^{1}$, Chien-Jung Lu ${ }^{1,2}$ and Chung-Yi Li ${ }^{5,6,7, * \mathbb{D}}$
}

1 Department of Neurology, En Chu Kong Hospital, New Taipei City 237, Taiwan;

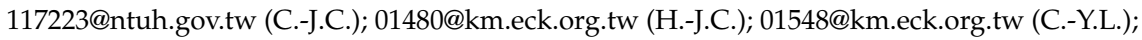
00520@km.eck.org.tw (C.-H.L.); 00202@km.eck.org.tw (C.-H.L.); 01181@km.eck.org.tw (C.-J.L.)

2 Department of Neurology, National Taiwan University Hospital, Taipei 100, Taiwan

3 Division of Pediatric Infectious Diseases, Department of Pediatrics, National Taiwan University Hospital, Taipei 100, Taiwan; cylu@ntu.edu.tw

4 College of Medicine, National Taiwan University, Taipei 100, Taiwan

5 Department of Public Health, College of Medicine, National Cheng Kung University, Tainan 701, Taiwan; t88071016@gs.ncku.edu.tw

6 Department of Public Health, College of Public Health, China Medical University, Taichung 404, Taiwan

7 Department of Healthcare Administration, College of Medical and Health Science, Asia University, Taichung 413, Taiwan

* Correspondence: 00034@km.eck.org.tw (Y.S.); cyli99@mail.ncku.edu.tw (C.-Y.L.)

\section{check for}

updates

Citation: Cheng, C.-J.; Lu, C.-Y.; Chang, Y.-H.; Sun, Y.; Chu, H.-J.; Lee, C.-Y.; Liu, C.-H.; Lin, C.-H.; Lu, C.-J.;

$\mathrm{Li}, \mathrm{C}$.-Y. Effectiveness of the WHO-Authorized COVID-19 Vaccines: A Rapid Review of Global Reports till 30 June 2021. Vaccines 2021, 9, 1489. https://doi.org/ $10.3390 /$ vaccines 9121489

Academic Editor:

Alessandra Casuccio

Received: 13 November 2021 Accepted: 13 December 2021 Published: 16 December 2021

Publisher's Note: MDPI stays neutral with regard to jurisdictional claims in published maps and institutional affiliations.

Copyright: (c) 2021 by the authors. Licensee MDPI, Basel, Switzerland. This article is an open access article distributed under the terms and conditions of the Creative Commons Attribution (CC BY) license (https:// creativecommons.org/licenses/by/ $4.0 /)$.
Abstract: Large clinical trials have proven the efficacy of the COVID-19 vaccine, and the number of studies about the effectiveness rapidly grew in the first half of the year after mass vaccination was administrated globally. This rapid review aims to provide evidence syntheses as a means to complement the current evidence on the vaccine effectiveness (VE) against various outcomes in real-world settings. Databases (PubMed, EMBASE, and MedRxiv) were searched up to 30 June 2021, (PROSPERO ID: 266866). A total of 39 studies were included, covering over 15 million participants from 11 nations. Among the general population being fully vaccinated, the VE against symptomatic SARS-CoV-2 infection was estimated at $89-97 \%$, 92\% (95\% CI, 78-97\%), and 94\% (95\% CI, 86-97\%) for BNT162b2, ChAdOx1, and mRNA-1273, respectively. As for the protective effects against B.1.617.2related symptomatic infection, the VE was $88 \%$ (95\% CI, 85.3-90.1\%) by BNT162b2 and $67.0 \%$ (95\% CI, $61.3-71.8 \%$ ) by ChAdOx1 after full vaccination. This review revealed a consistently high effectiveness of certain vaccines among the general population in real-world settings. However, scarce data on the major variants of SARS-CoV-2 and the shortness of the study time may limit the conclusions to the mRNA vaccines and ChAdOx1.

Keywords: COVID-19; COVID-19 vaccines; SARS-CoV-2 variants

\section{Introduction}

Large randomized control trials have demonstrated the efficacy against SARS-CoV2 infection to be $70 \%, 94 \%, 95 \%, 78 \%$, and $84 \%$ after two doses of the ChAdOx1 (Oxford/AstraZeneca, Oxford, UK), mRNA-1273 (Moderna, Cambridge, MA, USA), BNT162b2 (BioNTech/Pfizer, Mainz, Germany), BBIBP-CorV (Sinopharm, Beijing, China), and CoronaVac (Sinovac, Beijing, China) vaccines, respectively [1-5]. A single dose of the Ad26.COV2.S (Johnson \& Johnson, New Brunswick, NJ, USA) vaccine yielded an efficacy of $82 \%$ against severe-critical COVID-19 disease [6]. Based on the good results from clinical trials, COVID19 vaccination programs have been extensively rolled out in many countries around the world. However, over the first half-year since vaccine administration globally, most countries have full vaccination rates of less than $50 \%$ [7]. Billions of people in the world are eagerly waiting for the COVID-19 vaccines on the one hand and questioning how well the vaccines work in the real world on the other hand. As relevant study reports have been 
released successively, a wide range of vaccine effectiveness (VE) has been noticed. The estimated effectiveness after full vaccination could range from 50\% [8] to $100 \%$ [9], according to effectiveness studies of the six vaccines (BNT162b2, ChAdOx1, Ad26.COV2.S, mRNA-1273, BBIBP-CorV, CoronaVac), which were listed on the World Health Organization (WHO) Emergency Use Listing as of June 2021 [10].

Given a different efficacy was shown for vaccines developed with different platforms [1-4], their effectiveness in the real world for different populations needs to be confirmed. A Denmark study showed different VE in different age groups $(77 \%$ for people $\geq 85$ years of age vs. $86 \%$ for people $\geq 65$ years), and different living/working environments ( $53 \%$ for long-term care facilities dwellers vs. $80 \%$ for healthcare workers) [11]. Similarly, the VE may also be different among different countries or races. Attention should also be paid when VE from different studies are compared as VE of a given vaccine depends on what outcomes were chosen in determining VE. A study on the residents of long-term care facilities in Spain showed the VE of mRNA vaccines was $70 \%$ against asymptomatic infection and $97 \%$ against death [12]. In addition, many SARS-CoV-2 variants have been evolving since the pandemic. B.1.1.7 (alpha), B.1.351 (beta), B.1.617.2 (delta), and P.1 (gamma) variants, which are circulating in the whole world and are causing serious infections and mortality rate that are classified as variants of concern (VOC) by WHO. The VE against these VOC needs to be explored from the literature.

This rapid review aimed to assess the effectiveness of WHO-authorized COVID-19 vaccines, taking into account the aforementioned factors including country, characteristics of the study population, study design, outcomes, and the analysis of the involved VOC.

\section{Materials and Methods}

This rapid review was conducted based on the updated guideline of PRISMA 2020 statement and its recommended checklist $[13,14]$. We registered this review on PROSPERO (ID: 266866) on 13 July 2021. The eligibility criteria are reports evaluating the effectiveness of COVID-19 vaccines in populations aged $\geq 16$ years. Additional inclusion criteria require that the sample size of the vaccinated population should be more than 1000 to have a sufficient event number. Two authors (C.-J.C., Y.S.) performed the literature search in PubMed, EMBASE, and medRxiv. The search terms in PubMed and EMBASE were "effectiveness", "COVID-19 vaccine", and publish time "2021". Preprint articles from medRxiv were searched with the terms "effectiveness COVID-19 vaccine" or "effectiveness SARS-CoV-2 vaccine" in the titles or abstracts.

The article types we reviewed included original investigations, research letters, short communications, and correspondence articles. While screening the title and abstracts of the relevant articles in PubMed, similar research with titles shown on the web page was also checked. We updated our search up to 30 June 2021. C.J.C. and Y.S. contributed to the title and abstract screening for relevance and reviewing of full-text articles against inclusion and exclusion criteria. We excluded the following research: in vitro studies, animal studies, experimental clinical trials, systematic reviews or meta-analyses, diagnostic studies, methodological publications, editorial-style reviews, abstracts of posters, secondary analyses, studies with only immunogenicity data, safety reports or post-infection treatment, and articles with analyses only on a very specific target population, such as veterans, dentists, pregnant women, and patients with malignancy or mental illness.

Ethical approval was not applicable because all the study materials of this review are from the published articles and preprints.

After identification of all relevant articles, quality assessment was performed based on ROBINS-I of Cochrane Handbook to assess the risk of bias. Each bias domain and overall risk of bias was judged as "Low", "Moderate", "Serious", or "Critical" risk of bias based on the check list on the ROBINS-I assessment chart. The extracted data included the following items: author, country, number of vaccinated and unvaccinated participants, study design, age and characteristic of participants, types of vaccine, outcomes, definition about minimal intervals between vaccination (first dose and second dose), and event 
measurement, involvement of SARS-CoV-2 VOC, and VE with confidence interval (CI) respectively after first dose and second dose of vaccine.

The formula for calculating VE is ( 1 - hazard ratio for SARS-CoV-2 infection in vaccinated vs. unvaccinated participants) $\times 100 \%$. In studies that reported the incidence of infection, we calculated the incidence rate ratio (IRR) and converted it to unadjusted VE as $(1-$ IRR in vaccinated vs. unvaccinated participants $) \times 100 \%$. The VE from a case-control study was calculated as $(1-$ odds ratio $) \times 100 \%$. In case of insufficient data in an article, we contacted the authors to obtain the required information by email. We applied narrative synthesis to process the data from the included studies. As the number of studies of VE constantly grew over our processing period, this review only included reports that were released before 1 July 2021. Distributions of the VE estimates derived from the included studies were further graphically presented by Box plots, according to the study population, brand of vaccine, variant, number of doses, and outcome.

\section{Results}

\subsection{Study Selection and Characteristics}

Of 2369 searched articles (2085 from PubMed, 195 from EMBASE, and 89 preprints from MedRxiv), 2312 were excluded while screening the abstracts and titles. After a full-text review of the remaining 57 articles for eligibility, 18 were removed due to not completely meeting the criteria. Therefore, 39 studies $[9,11,12,15-50]$ that met the inclusion criteria were included in this rapid review (Figure 1, Supplementary Table S1). The results of the quality assessment of all the included studies are shown in Supplementary Table S2. Before 1 July 2021,24 of 39 studies were published in peer-reviewed journals $[9,12,15-17,19,22,24,25,28$ $31,33,34,36,37,40-42,45,47,48,50$ ] while the remaining 15 studies were posted online as preprint articles $[11,18,20,21,23,26,27,32,35,38,39,43,44,46,49]$. The characteristics of the studies are shown in Supplementary Table S1, including the country, study design, types of vaccines, outcomes, and SARS-CoV-2 variants involved in the studies. The outcomes include all laboratory-confirmed SARS-CoV-2 infection, asymptomatic and symptomatic infection, hospitalization, critical disease, and death. As for evaluating the protective effects of the vaccine on SARS-CoV-2 variants, 5 studies mentioned the approximate prevalence of variants in the region of the study population $[18,19,28,34,49]$, and 11 studies calculated the number or percentage of cases with variants among all or sampled participants with a positive test of SARS-CoV-2 variants [20,26,31,33,36-38,42,43,46,48]. Only three studies evaluated the VE against specific VOC, in which one study from Qatar reported the VE against B.1.1.7 and B.1.351 [9], one study from Canada studied VE against B.1.1.7 and P.1 [46], and the other one study from the UK evaluated the VE against B.1.1.7 and B.1.617.2 (delta variant) [20]. 


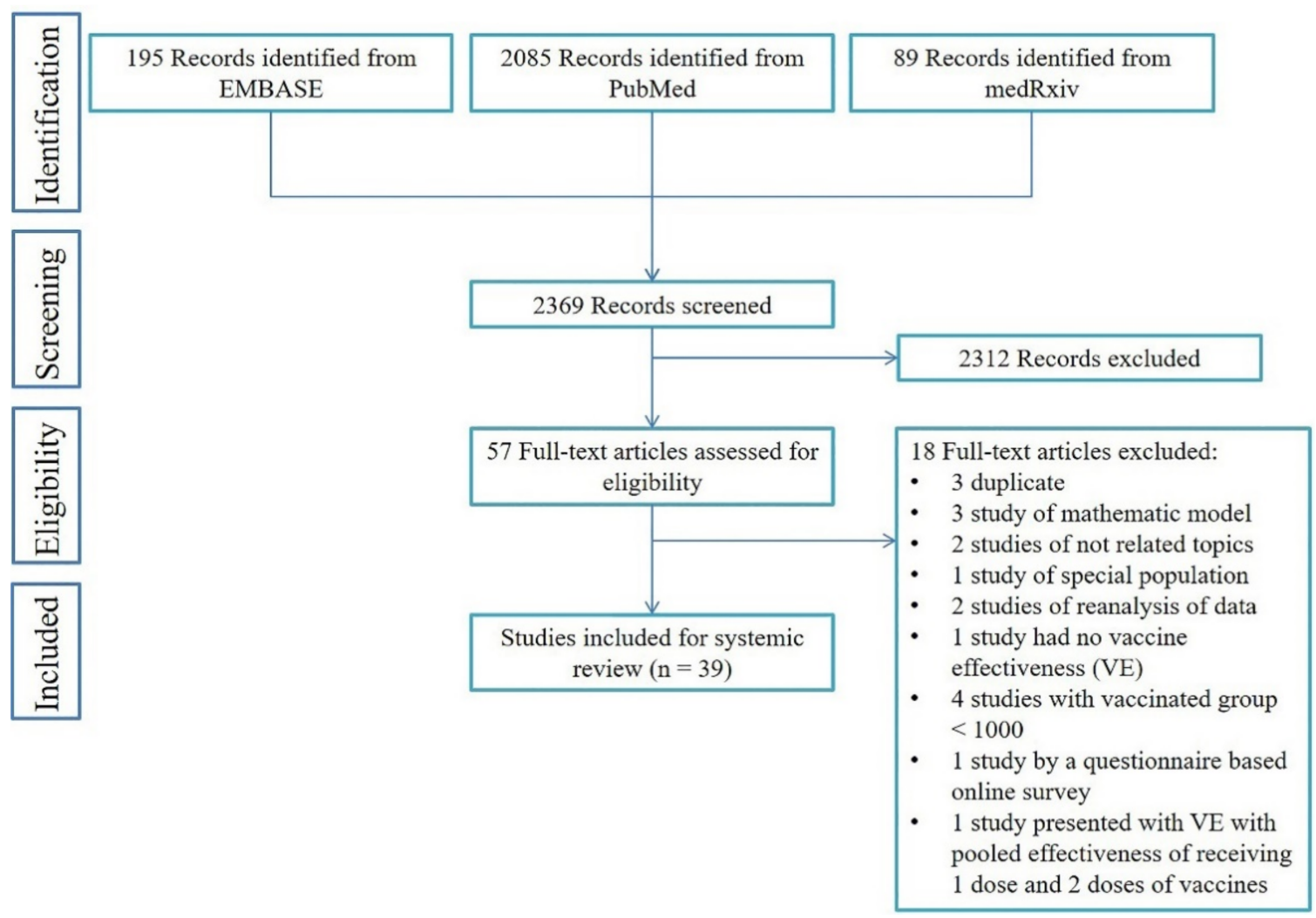

Figure 1. PRISMA flowchart of the literature searches.

3.2. Effectiveness after Partial or Full Vaccination for Various Outcome of Interest

3.2.1. Overall SARS-CoV-2 Infection across General and Sub-Populations

The characteristics of the studies and their VE estimates with $95 \% \mathrm{CI}$ against overall SARS-CoV-2 infection among general and specific populations are shown in Table 1 and the distributions of the VE estimates are shown in Figure 2. Among the partially vaccinated general population, the VE varied among studies (42-78\% by BNT162b2 [23,28,31,32,40,42,46], 67-93\% by mRNA-1273 $[26,31,40]$, and $61-95 \%$ by ChAdOx1 [31,32,42]). After full vaccination, the VE was all higher than $75 \%$ (77-98\% by BNT162b2 [11,23,28,31,33,40,42], $93-99 \%$ by mRNA-1273 [31,40], 79\% by ChAdOx1 [42], and 78\% by Ad26.COV2.S with a single dose as full vaccination [27]). The only exception was a low VE noted for CoronaVac (42\%, $95 \%$ CI, 26.9-53.3\%) in a Brazilian study of subjects aged $\geq 70$, with an $83 \%$ P.1 variant prevalence during the study time (Table 1) [43]. The VE among fully vaccinated healthcare workers was $80 \%$ or higher $(80-97 \%$ by BNT162b2 [11,19,22,30,34,39,47,48] and $82-99 \%$ by mRNA-1273 [47,48]) (Table 1). Comparatively, VE estimates among residents of long-term care facilities were low (partially vaccinated: $21-65 \%$ by BNT162b2 [39,45], 68\% [95\% CI, $34-85 \%$ ] by ChAdOx1 [45]; fully vaccinated: $53-64 \%$ by BNT162b2 [11,39]). Additionally, the VE was also relatively low among subjects with comorbidity or chronic illness (one dose of ChAdOx1: $24 \%$ after $\geq 21$ days, $50 \%$ after $\geq 42$ days [18], two doses of BNT162b2: $71 \%$ after $\geq 7$ days [11]) (Table 1 ). 
Table 1. Summary of the studies on the effectiveness of COVID-19 vaccines.

\begin{tabular}{|c|c|c|c|c|c|c|c|c|c|c|}
\hline \multicolumn{11}{|c|}{ The Effectiveness of COVID-19 Vaccines among General Population } \\
\hline $\begin{array}{c}\text { First } \\
\text { Author/Country }\end{array}$ & Study Design & $\begin{array}{l}\text { No. of Vaccinated/No. } \\
\text { of Unvaccinated }\end{array}$ & Age (Years) & Vaccine & Outcomes & $\begin{array}{l}\text { Days after the } \\
\text { 1st Dose }\end{array}$ & $\begin{array}{l}\text { VE of 1st Dose } \\
\quad(95 \% \mathrm{CI})\end{array}$ & $\begin{array}{l}\text { Days after the } \\
\text { 2nd Dose }\end{array}$ & $\begin{array}{l}\text { VE of 2nd Dose } \\
(95 \% \mathrm{CI})\end{array}$ & $\begin{array}{l}\text { Variants } \\
\text { Involved }\end{array}$ \\
\hline \multirow[t]{5}{*}{$\begin{array}{l}\text { Dagan et al./ } \\
\text { Israel [28] }\end{array}$} & \multirow[t]{5}{*}{ Cohort study } & $596,618 / 596,618$ & $\geq 16$ & BNT162b2 & Overall infection & $14-20$ & $\begin{array}{c}46 \% \\
(40-51 \%)\end{array}$ & $\geq 7$ & $\begin{array}{c}92 \% \\
(88-95 \%)\end{array}$ & B.1.1.7 \\
\hline & & $596,618 / 596,618$ & $\geq 16$ & BNT162b2 & $\begin{array}{l}\text { Symptomatic } \\
\text { infection }\end{array}$ & $14-20$ & $\begin{array}{c}57 \% \\
(50-63 \%)\end{array}$ & $\geq 7$ & $\begin{array}{c}94 \% \\
(87-98 \%)\end{array}$ & B.1.1.7 \\
\hline & & $596,618 / 596,618$ & $\geq 16$ & BNT162b2 & Hospitalization & $14-20$ & $\begin{array}{c}74 \% \\
(56-86 \%)\end{array}$ & $\geq 7$ & $\begin{array}{c}87 \% \\
(55-100 \%)\end{array}$ & B.1.1.7 \\
\hline & & $596,618 / 596,618$ & $\geq 16$ & BNT162b2 & Critical disease & $14-20$ & $\begin{array}{c}62 \% \\
(39-80 \%)\end{array}$ & $\geq 7$ & $\begin{array}{c}92 \% \\
(75-100 \%)\end{array}$ & B.1.1.7 \\
\hline & & $596,618 / 596,618$ & $\geq 16$ & BNT162b2 & Death & $14-20$ & $\begin{array}{c}72 \% \\
(19-100 \%) \\
\end{array}$ & $\mathrm{N} / \mathrm{A}$ & N/A & B.1.1.7 \\
\hline \multirow[t]{5}{*}{$\begin{array}{l}\text { Haas et al./ } \\
\text { Israel [33] }\end{array}$} & \multirow[t]{5}{*}{ Cohort study } & $4,714,932 / 1,823,979^{a}$ & $\geq 16$ & BNT162b2 & Overall infection & $\mathrm{N} / \mathrm{A}$ & $\mathrm{N} / \mathrm{A}$ & $\geq 7$ & $\begin{array}{c}95.3 \% \\
(94.9-95.7 \%)\end{array}$ & B.1.1.7 \\
\hline & & $4,714,932 / 1,823,979^{a}$ & $\geq 16$ & BNT162b2 & $\begin{array}{l}\text { Asymptomatic } \\
\text { infection }\end{array}$ & $\mathrm{N} / \mathrm{A}$ & $\mathrm{N} / \mathrm{A}$ & $\geq 7$ & $\begin{array}{c}91.5 \% \\
(90.7-92.2 \%)\end{array}$ & B.1.1.7 \\
\hline & & $4,714,932 / 1,823,979^{\text {a }}$ & $\geq 16$ & BNT162b2 & $\begin{array}{l}\text { Symptomatic } \\
\text { infection }\end{array}$ & $\mathrm{N} / \mathrm{A}$ & $\mathrm{N} / \mathrm{A}$ & $\geq 7$ & $\begin{array}{c}97.0 \% \\
(96.7-97.2 \%)\end{array}$ & B.1.1.7 \\
\hline & & $4,714,932 / 1,823,979^{\text {a }}$ & $\geq 16$ & BNT162b2 & Hospitalization & $\mathrm{N} / \mathrm{A}$ & $\mathrm{N} / \mathrm{A}$ & $\geq 7$ & $\begin{array}{c}97.2 \% \\
(96.8-97.5 \%)\end{array}$ & B.1.1.7 \\
\hline & & $4,714,932 / 1,823,979^{\mathrm{a}}$ & $\geq 16$ & BNT162b2 & Death & $\mathrm{N} / \mathrm{A}$ & $\mathrm{N} / \mathrm{A}$ & $\geq 7$ & $\begin{array}{c}96.7 \% \\
(96.0-97.3 \%) \\
\end{array}$ & B.1.1.7 \\
\hline \multirow[t]{6}{*}{$\begin{array}{l}\text { Pritchard et al./ } \\
\text { UK [42] }\end{array}$} & \multirow[t]{6}{*}{$\begin{array}{l}\text { Case-control } \\
\text { study }\end{array}$} & $67,738 / 192,224$ & $\geq 16$ & BNT162b2 & Overall infection & $\geq 21$ & $\begin{array}{c}66 \% \\
(60-71 \%)\end{array}$ & $\geq 1$ & $\begin{array}{c}80 \% \\
(73-85 \%)\end{array}$ & B.1.1.7 \\
\hline & & $123,850 / 192,224$ & $\geq 16$ & ChAdOx1 & Overall infection & $\geq 21$ & $\begin{array}{c}61 \% \\
(54-68 \%)\end{array}$ & $\geq 1$ & $\begin{array}{c}79 \% \\
(65-88 \%)\end{array}$ & B.1.1.7 \\
\hline & & $67,738 / 192,224$ & $\geq 16$ & BNT162b2 & $\begin{array}{l}\text { Asymptomatic } \\
\text { infection }\end{array}$ & $\geq 21$ & $\begin{array}{c}47 \% \\
(35-57 \%)\end{array}$ & $\geq 1$ & $\begin{array}{c}58 \% \\
(43-69 \%)\end{array}$ & B.1.1.7 \\
\hline & & $123,850 / 192,224$ & $\geq 16$ & ChAdOx1 & $\begin{array}{l}\text { Asymptomatic } \\
\text { infection }\end{array}$ & $\geq 21$ & $\begin{array}{c}47 \% \\
(33-58 \%)\end{array}$ & $\geq 1$ & $\begin{array}{c}61 \% \\
(27-79 \%)\end{array}$ & B.1.1.7 \\
\hline & & $67,738 / 192,224$ & $\geq 16$ & BNT162b2 & $\begin{array}{l}\text { Symptomatic } \\
\text { infection }\end{array}$ & $\geq 21$ & $\begin{array}{c}78 \% \\
(72-83 \%)\end{array}$ & $\geq 1$ & $\begin{array}{c}95 \% \\
(91-98 \%)\end{array}$ & B.1.1.7 \\
\hline & & $123,850 / 192,224$ & $\geq 16$ & ChAdOx1 & $\begin{array}{l}\text { Symptomatic } \\
\text { infection }\end{array}$ & $\geq 21$ & $\begin{array}{c}71 \% \\
(62-78 \%)\end{array}$ & $\geq 1$ & $\begin{array}{c}92 \% \\
(78-97 \%)\end{array}$ & B.1.1.7 \\
\hline
\end{tabular}


Table 1. Cont

\begin{tabular}{|c|c|c|c|c|c|c|c|c|c|c|}
\hline \multicolumn{11}{|c|}{ The Effectiveness of COVID-19 Vaccines among General Population } \\
\hline $\begin{array}{c}\text { First } \\
\text { Author/Country }\end{array}$ & Study Design & $\begin{array}{l}\text { No. of Vaccinated/No. } \\
\text { of Unvaccinated }\end{array}$ & Age (Years) & Vaccine & Outcomes & $\begin{array}{l}\text { Days after the } \\
\text { 1st Dose }\end{array}$ & $\begin{array}{l}\text { VE of 1st Dose } \\
(95 \% \mathrm{CI})\end{array}$ & $\begin{array}{l}\text { Days after the } \\
\text { 2nd Dose }\end{array}$ & $\begin{array}{l}\text { VE of 2nd Dose } \\
(95 \% \mathrm{CI})\end{array}$ & $\begin{array}{l}\text { Variants } \\
\text { Involved }\end{array}$ \\
\hline \multirow[t]{6}{*}{$\begin{array}{c}\text { Pawlowski et al./ } \\
\text { US [40] }\end{array}$} & \multirow[t]{6}{*}{ Cohort study } & $51,795 / 51,795$ & $\geq 18$ & BNT162b2 & Overall infection & $\geq 14$ & $\begin{array}{c}61.0 \% \\
(50.8-69.2 \%)\end{array}$ & $\geq 7$ & $\begin{array}{c}86.1 \% \\
(82.4-89.1 \%)\end{array}$ & \\
\hline & & $16,471 / 16,471$ & $\geq 18$ & mRNA-1273 & Overall infection & $\geq 14$ & $\begin{array}{c}66.6 \% \\
(51.9-77.3 \%)\end{array}$ & $\geq 7$ & $\begin{array}{c}93.3 \% \\
(85.7-97.4 \%)\end{array}$ & \\
\hline & & $51,795 / 51,795$ & $\geq 18$ & BNT162b2 & Hospitalization & $\mathrm{N} / \mathrm{A}$ & $\mathrm{N} / \mathrm{A}$ & $\geq 7$ & $\begin{array}{c}88.8 \% \\
(75.5-95.7 \%)\end{array}$ & \\
\hline & & $16,471 / 16,471$ & $\geq 18$ & mRNA-1273 & Hospitalization & $\mathrm{N} / \mathrm{A}$ & $\mathrm{N} / \mathrm{A}$ & $\geq 7$ & $\begin{array}{c}86.0 \% \\
(71.6-93.9 \%)\end{array}$ & \\
\hline & & $51,795 / 51,795$ & $\geq 18$ & BNT162b2 & ICU admission & $\mathrm{N} / \mathrm{A}$ & $\mathrm{N} / \mathrm{A}$ & $\geq 7$ & $\begin{array}{c}100.0 \% \\
(51.4-100 \%)\end{array}$ & \\
\hline & & $16,471 / 16,471$ & $\geq 18$ & mRNA-1273 & ICU admission & $\mathrm{N} / \mathrm{A}$ & $\mathrm{N} / \mathrm{A}$ & $\geq 7$ & $\begin{array}{c}100.0 \% \\
(43.3-100 \%)\end{array}$ & \\
\hline $\begin{array}{c}\text { Björk et al./ } \\
\text { Sweden [23] }^{\mathrm{b}}\end{array}$ & Cohort study & $26,587 / 779,154$ & $18-64$ & BNT162b2 & Overall infection & $\geq 14$ & $\begin{array}{c}42 \% \\
(14-63 \%)\end{array}$ & $\geq 7$ & $\begin{array}{c}86 \% \\
(72-94 \%)\end{array}$ & \\
\hline \multirow[t]{4}{*}{$\begin{array}{c}\text { Abu-Raddad et al./ } \\
\text { Qatar [9] }\end{array}$} & \multirow[t]{4}{*}{$\begin{array}{l}\text { Case-control } \\
\text { study }\end{array}$} & $51,324 / 162,434$ & N/A & BNT162b2 & Infection of B.1.1.7 & $\geq 1$ & $\begin{array}{c}29.5 \% \\
(22.9-35.5 \%)\end{array}$ & $\geq 14$ & $\begin{array}{c}89.5 \% \\
(85.9-92.3 \%)\end{array}$ & B.1.1.7 \\
\hline & & $51,324 / 162,434$ & $\mathrm{~N} / \mathrm{A}$ & BNT162b2 & Infection of B.1.351 & $\geq 1$ & $\begin{array}{c}16.9 \% \\
(10.4-23.0 \%)\end{array}$ & $\geq 14$ & $\begin{array}{c}75.0 \% \\
(70.5-78.9 \%)\end{array}$ & B.1.351 \\
\hline & & $51,324 / 162,434$ & N/A & BNT162b2 & $\begin{array}{c}\text { fatal disease caused } \\
\text { by the B.1.351 } \\
\text { variant }\end{array}$ & $\geq 1$ & $\begin{array}{c}0.0 \% \\
(0.0-19.0 \%)\end{array}$ & $\geq 14$ & $\begin{array}{c}100.0 \% \\
(73.7-100.0 \%)\end{array}$ & \multirow[t]{2}{*}{ B.1.351 } \\
\hline & & $51,324 / 162,434$ & N/A & BNT162b2 & $\begin{array}{c}\text { Severe, critical, or } \\
\text { fatal disease caused } \\
\text { by any SARS-CoV-2 }\end{array}$ & $\geq 1$ & $\begin{array}{c}39.4 \% \\
(24.0-51.8 \%)\end{array}$ & $\geq 14$ & $\begin{array}{c}97.4 \% \\
(92.2-99.5 \%)\end{array}$ & \\
\hline
\end{tabular}


Table 1. Cont

\begin{tabular}{|c|c|c|c|c|c|c|c|c|c|c|}
\hline \multicolumn{11}{|c|}{ The Effectiveness of COVID-19 Vaccines among General Population } \\
\hline $\begin{array}{c}\text { First } \\
\text { Author/Country }\end{array}$ & Study Design & $\begin{array}{l}\text { No. of Vaccinated/No. } \\
\text { of Unvaccinated }\end{array}$ & Age (Years) & Vaccine & Outcomes & $\begin{array}{l}\text { Days after the } \\
\text { 1st Dose }\end{array}$ & $\begin{array}{l}\text { VE of 1st Dose } \\
\text { (95\% CI) }\end{array}$ & $\begin{array}{l}\text { Days after the } \\
\text { 2nd Dose }\end{array}$ & $\begin{array}{l}\text { VE of 2nd Dose } \\
(95 \% \text { CI })\end{array}$ & $\begin{array}{l}\text { Variants } \\
\text { Involved }\end{array}$ \\
\hline \multirow[t]{6}{*}{$\begin{array}{c}\text { Lopez Bernal et al./ } \\
\text { UK [46] }\end{array}$} & $\begin{array}{l}\text { Case-control } \\
\text { study }\end{array}$ & $5553 / 24,706$ & $\geq 80$ & BNT162b2 & $\begin{array}{l}\text { Symptomatic } \\
\text { infection }\end{array}$ & $28-34$ & $\begin{array}{c}70 \% \\
(59-78 \%)\end{array}$ & $\geq 14$ & $\begin{array}{c}89 \% \\
(85-93 \%)\end{array}$ & B.1.1.7 \\
\hline & & $12,122 / 51,955$ & $\geq 70$ & BNT162b2 & $\begin{array}{l}\text { Symptomatic } \\
\text { infection }\end{array}$ & $28-34$ & $\begin{array}{c}61 \% \\
(51-69 \%)\end{array}$ & $\mathrm{N} / \mathrm{A}$ & N/A & B.1.1.7 \\
\hline & & $10,544 / 51,955$ & $\geq 70$ & ChAdOx1 & $\begin{array}{l}\text { Symptomatic } \\
\text { infection }\end{array}$ & $28-34$ & $\begin{array}{c}60 \% \\
(41-73 \%)\end{array}$ & $\mathrm{N} / \mathrm{A}$ & $\mathrm{N} / \mathrm{A}$ & B.1.1.7 \\
\hline & & $3484 / 8892$ & $\geq 80$ & BNT162b2 & Hospitalization & $\geq 14$ & $\begin{array}{l}\text { Further } 43 \% \\
(33-52 \%)^{c}\end{array}$ & $\mathrm{~N} / \mathrm{A}$ & $\mathrm{N} / \mathrm{A}$ & B.1.1.7 \\
\hline & & $688 / 8892$ & $\geq 80$ & ChAdOx1 & Hospitalization & $\geq 14$ & $\begin{array}{l}\text { Further } 37 \% \\
(3-59 \%)^{\mathrm{c}}\end{array}$ & $\mathrm{N} / \mathrm{A}$ & $\mathrm{N} / \mathrm{A}$ & B.1.1.7 \\
\hline & & $1846 / 8096$ & $\geq 80$ & BNT162b2 & Death & $\geq 14$ & $\begin{array}{l}\text { Further } 51 \% \\
(37-62 \%)^{c}\end{array}$ & $\mathrm{~N} / \mathrm{A}$ & $\mathrm{N} / \mathrm{A}$ & B.1.1.7 \\
\hline \multirow[t]{3}{*}{$\begin{array}{l}\text { Vasileiou et al./ } \\
\text { UK [50] }\end{array}$} & Cohort study & $1,331,993 / 3,077,595$ & $\geq 18$ & $\begin{array}{l}\text { BNT162b2, } \\
\text { ChAdOx1 }\end{array}$ & Hospitalization & $28-34$ & $\begin{array}{c}89 \% \\
(83-92 \%)\end{array}$ & $\mathrm{N} / \mathrm{A}$ & $\mathrm{N} / \mathrm{A}$ & \\
\hline & & $711,839 / 3,077,595$ & $\geq 18$ & BNT162b2 & Hospitalization & $28-34$ & $\begin{array}{c}91 \% \\
(85-94 \%)\end{array}$ & $\mathrm{N} / \mathrm{A}$ & $\mathrm{N} / \mathrm{A}$ & \\
\hline & & $620,154 / 3,077,595$ & $\geq 18$ & ChAdOx1 & Hospitalization & $28-34$ & $\begin{array}{c}88 \% \\
(75-94 \%)\end{array}$ & $\mathrm{N} / \mathrm{A}$ & $\mathrm{N} / \mathrm{A}$ & \\
\hline $\begin{array}{l}\text { Glampson et al./ } \\
\text { UK [32] }{ }^{\mathrm{b}}\end{array}$ & & $163,452 / 1,797,286$ & $\geq 16$ & ChAdOx1 & Overall infection & 28 & $\begin{array}{c}74 \% \\
(65-81 \%) \\
\end{array}$ & $\mathrm{N} / \mathrm{A}$ & $\mathrm{N} / \mathrm{A}$ & \\
\hline $\begin{array}{l}\text { Corchado-Garcia } \\
\text { et al./US [27] }{ }^{\mathrm{b}}\end{array}$ & Cohort study & $2195 / 21,950$ & $\geq 18$ & Ad26.COV2.S & Overall infection & $\geq 14$ & $\begin{array}{c}76.7 \% \\
(30.3-95.3 \%) \\
\end{array}$ & $\mathrm{N} / \mathrm{A}$ & $\mathrm{N} / \mathrm{A}$ & \\
\hline \multirow[t]{2}{*}{$\begin{array}{l}\text { Lopez Bernal et al./ } \\
\text { UK [21] }^{\mathrm{b}}\end{array}$} & Cohort study & $6108 / 38,038$ & $\geq 70$ & BNT162b2 & Death & $\geq 21$ & $\begin{array}{l}\text { Further } 44 \% \\
(32-53 \%)^{\mathrm{c}}\end{array}$ & $\geq 7$ & $\begin{array}{l}\text { Further 69\% } \\
(31-86 \%)\end{array}$ & \\
\hline & & $3950 / 38,038$ & $\geq 70$ & ChAdOx1 & Death & $\geq 21$ & $\begin{array}{l}\text { Further 55\% } \\
(41-66 \%)^{c}\end{array}$ & $\mathrm{~N} / \mathrm{A}$ & $\mathrm{N} / \mathrm{A}$ & \\
\hline
\end{tabular}


Table 1. Cont

\begin{tabular}{|c|c|c|c|c|c|c|c|c|c|c|}
\hline \multicolumn{11}{|c|}{ The Effectiveness of COVID-19 Vaccines among General Population } \\
\hline $\begin{array}{c}\text { First } \\
\text { Author/Country }\end{array}$ & Study Design & $\begin{array}{l}\text { No. of Vaccinated/No. } \\
\text { of Unvaccinated }\end{array}$ & Age (Years) & Vaccine & Outcomes & $\begin{array}{l}\text { Days after the } \\
\text { 1st Dose }\end{array}$ & $\begin{array}{l}\text { VE of 1st Dose } \\
\text { (95\% CI) }\end{array}$ & $\begin{array}{l}\text { Days after the } \\
\text { 2nd Dose }\end{array}$ & $\begin{array}{l}\text { VE of 2nd Dose } \\
(95 \% \mathrm{CI})\end{array}$ & $\begin{array}{l}\text { Variants } \\
\text { Involved }\end{array}$ \\
\hline \multirow[t]{9}{*}{$\begin{array}{l}\text { Chung et al./ } \\
\text { Canada [26] }\end{array}$} & \multirow[t]{9}{*}{$\begin{array}{l}\text { Case-control } \\
\text { study }\end{array}$} & $21,272 / 302,761$ & $\geq 16$ & $\begin{array}{l}\text { BNT162b2, } \\
\text { mRNA-1273 }\end{array}$ & $\begin{array}{l}\text { Symptomatic } \\
\text { infection }\end{array}$ & $\geq 14$ & $\begin{array}{c}60 \% \\
(57-64 \%)\end{array}$ & $\geq 7$ & $\begin{array}{c}91 \% \\
(89-93 \%)\end{array}$ & \\
\hline & & $18,332 / 302,761$ & $\geq 16$ & BNT162b2 & $\begin{array}{l}\text { Symptomatic } \\
\text { infection }\end{array}$ & $\geq 14$ & $\begin{array}{c}59 \% \\
(55-62 \%)\end{array}$ & $\geq 7$ & $\begin{array}{c}91 \% \\
(88-93 \%)\end{array}$ & \\
\hline & & $2940 / 302,761$ & $\geq 16$ & mRNA-1273 & $\begin{array}{c}\text { Symptomatic } \\
\text { infection }\end{array}$ & $\geq 14$ & $\begin{array}{c}72 \% \\
(63-80 \%)\end{array}$ & $\geq 7$ & $\begin{array}{c}94 \% \\
(86-97 \%)\end{array}$ & \\
\hline & & $21,272 / 302,761$ & $\geq 16$ & $\begin{array}{l}\text { BNT162b2, } \\
\text { mRNA-1273 }\end{array}$ & $\begin{array}{l}\text { Hospitalization, or } \\
\text { death }\end{array}$ & $\geq 14$ & $\begin{array}{c}70 \% \\
(60-77 \%)\end{array}$ & $\geq 7$ & $\begin{array}{c}98 \% \\
(88-100 \%)\end{array}$ & \\
\hline & & $18,332 / 302,761$ & $\geq 16$ & BNT162b2 & $\begin{array}{c}\text { Hospitalization, or } \\
\text { death }\end{array}$ & $\geq 14$ & $\begin{array}{c}69 \% \\
(59-77 \%)\end{array}$ & $\geq 0$ & $\begin{array}{c}96 \% \\
(82-99 \%)\end{array}$ & \\
\hline & & $2940 / 302,761$ & $\geq 16$ & mRNA-1273 & $\begin{array}{l}\text { Hospitalization, or } \\
\text { death }\end{array}$ & $\geq 14$ & $\begin{array}{c}73 \% \\
(42-87 \%)\end{array}$ & $\geq 0$ & $\begin{array}{c}96 \% \\
(74-100 \%)\end{array}$ & \\
\hline & & $21,272 / 302,761$ & $\geq 16$ & $\begin{array}{l}\text { BNT162b2, } \\
\text { mRNA-1273 }\end{array}$ & $\begin{array}{c}\text { Symptomatic } \\
\text { infection of B.1.1.7 }\end{array}$ & $\geq 14$ & $\begin{array}{c}61 \% \\
(56-66 \%)\end{array}$ & $\geq 7$ & $\begin{array}{c}90 \% \\
(85-94 \%)\end{array}$ & B.1.1.7 \\
\hline & & $21,272 / 302,761$ & $\geq 16$ & $\begin{array}{l}\text { BNT162b2, } \\
\text { mRNA-1273 }\end{array}$ & $\begin{array}{c}\text { Symptomatic } \\
\text { infection of B.1.351 } \\
\text { or P.1 }\end{array}$ & $\geq 14$ & $\begin{array}{c}43 \% \\
(22-59 \%)\end{array}$ & $\geq 7$ & $\begin{array}{c}88 \% \\
(61-96 \%)\end{array}$ & B.1.351, P.1 \\
\hline & & $21,272 / 302,761$ & $\geq 16$ & $\begin{array}{l}\text { BNT162b2, } \\
\text { mRNA-1273 }\end{array}$ & $\begin{array}{c}\text { Hospitalization, or } \\
\text { death of B.1.1.7 }\end{array}$ & $\geq 14$ & $\begin{array}{c}59 \% \\
(39-73 \%)\end{array}$ & $\geq 0$ & $\begin{array}{c}94 \% \\
(59-99 \%)\end{array}$ & B.1.1.7 \\
\hline \multirow[t]{6}{*}{$\begin{array}{l}\text { Skowronski et al./ } \\
\text { Canada [46] }\end{array}$} & \multirow[t]{6}{*}{$\begin{array}{l}\text { Case-control } \\
\text { study }\end{array}$} & $12,471 / 4522$ & $\geq 70$ & $\begin{array}{l}\text { BNT162b2, } \\
\text { mRNA-1273 }\end{array}$ & Overall infection & $\geq 21$ & $\begin{array}{c}65 \% \\
(58-71 \%)\end{array}$ & $\mathrm{N} / \mathrm{A}$ & $\mathrm{N} / \mathrm{A}$ & \\
\hline & & $10,569 / 4522$ & $\geq 70$ & BNT162b2 & Overall infection & $\geq 21$ & $\begin{array}{c}64 \% \\
(57-71 \%)\end{array}$ & $\mathrm{N} / \mathrm{A}$ & $\mathrm{N} / \mathrm{A}$ & \\
\hline & & $1882 / 4522$ & $\geq 70$ & mRNA-1273 & Overall infection & $\geq 21$ & $\begin{array}{c}71 \% \\
(56-81 \%)\end{array}$ & N/A & N/A & \\
\hline & & $12,471 / 4522$ & $\geq 70$ & $\begin{array}{l}\text { BNT162b2, } \\
\text { mRNA-1273 }\end{array}$ & $\begin{array}{l}\text { Infection of } \\
\text { non-variant of } \\
\text { concern }\end{array}$ & $\geq 21$ & $\begin{array}{c}72 \% \\
(58-81 \%)\end{array}$ & $\mathrm{N} / \mathrm{A}$ & $\mathrm{N} / \mathrm{A}$ & $\begin{array}{l}\text { Non- } \\
\text { variant of } \\
\text { concern }\end{array}$ \\
\hline & & $12,471 / 4522$ & $\geq 70$ & $\begin{array}{l}\text { BNT162b2, } \\
\text { mRNA-1273 }\end{array}$ & Infection of B.1.1.7 & $\geq 21$ & $\begin{array}{c}67 \% \\
(57-75 \%)\end{array}$ & $\mathrm{N} / \mathrm{A}$ & $\mathrm{N} / \mathrm{A}$ & B.1.1.7 \\
\hline & & $12,471 / 4522$ & $\geq 70$ & $\begin{array}{l}\text { BNT162b2, } \\
\text { mRNA-1273 }\end{array}$ & Infection of P.1 & $\geq 21$ & $\begin{array}{c}61 \% \\
(45-72 \%)\end{array}$ & $\mathrm{N} / \mathrm{A}$ & $\mathrm{N} / \mathrm{A}$ & P.1 \\
\hline
\end{tabular}


Table 1. Cont.

\begin{tabular}{|c|c|c|c|c|c|c|c|c|c|c|}
\hline \multicolumn{11}{|c|}{ The Effectiveness of COVID-19 Vaccines among General Population } \\
\hline $\begin{array}{c}\text { First } \\
\text { Author/Country }\end{array}$ & Study Design & $\begin{array}{l}\text { No. of Vaccinated/No. } \\
\text { of Unvaccinated }\end{array}$ & Age (Years) & Vaccine & Outcomes & $\begin{array}{l}\text { Days after the } \\
\text { 1st Dose }\end{array}$ & $\begin{array}{l}\text { VE of 1st Dose } \\
\text { (95\% CI) }\end{array}$ & $\begin{array}{l}\text { Days after the } \\
\text { 2nd Dose }\end{array}$ & $\begin{array}{l}\text { VE of 2nd Dose } \\
\quad(95 \% \mathrm{CI})\end{array}$ & $\begin{array}{l}\text { Variants } \\
\text { Involved }\end{array}$ \\
\hline \multirow[t]{4}{*}{$\begin{array}{l}\text { Emborg et al./ } \\
\text { Denmark [11] }{ }^{\mathrm{b}}\end{array}$} & \multirow[t]{4}{*}{ Cohort study } & $473,957 / 390,139 \mathrm{~d}$ & \multirow{4}{*}{$\geq 85$} & BNT162b2 & Overall infection & $\mathrm{N} / \mathrm{A}$ & $\mathrm{N} / \mathrm{A}$ & 7 & $\begin{array}{c}82 \% \\
(79-84 \%)\end{array}$ & \\
\hline & & $79,185 / 19,348$ & & BNT162b2 & Overall infection & $\mathrm{N} / \mathrm{A}$ & N/A & 7 & $\begin{array}{c}77 \% \\
(50-89 \%)\end{array}$ & \\
\hline & & $473,957 / 390,139 \mathrm{~d}$ & & BNT162b2 & Hospitalization & $\mathrm{N} / \mathrm{A}$ & $\mathrm{N} / \mathrm{A}$ & 7 & $\begin{array}{c}93 \% \\
(89-96 \%)\end{array}$ & \\
\hline & & $473,957 / 390,139 \mathrm{~d}$ & & BNT162b2 & Death & $\mathrm{N} / \mathrm{A}$ & $\mathrm{N} / \mathrm{A}$ & 7 & $\begin{array}{c}94 \% \\
(90-96 \%)\end{array}$ & \\
\hline $\begin{array}{l}\text { Ranzani et al./ } \\
\text { Brazil [43] }^{\mathrm{b}}\end{array}$ & $\begin{array}{l}\text { Case-control } \\
\text { study }\end{array}$ & $4854 / 11,046$ & $\geq 70$ & CoronaVac & Infection & $\mathrm{N} / \mathrm{A}$ & $\mathrm{N} / \mathrm{A}$ & $\geq 14$ & $\begin{array}{c}41.6 \% \\
(26.9-53.3 \%)\end{array}$ & P.1 \\
\hline \multirow[t]{5}{*}{$\begin{array}{l}\text { Lopez Bernal et al./ } \\
\text { UK [20] }{ }^{\text {b }}\end{array}$} & \multirow[t]{5}{*}{$\begin{array}{l}\text { Case-control } \\
\text { study }\end{array}$} & $79,665 / 107,727$ & $\geq 16$ & $\begin{array}{l}\text { BNT162b2, } \\
\text { ChAdOx1 }\end{array}$ & $\begin{array}{c}\text { Symptomatic } \\
\text { infection of B.1.1.7 }\end{array}$ & $\geq 21$ & $\begin{array}{c}48.7 \% \\
(45.5-51.7 \%)\end{array}$ & $\geq 14$ & $\begin{array}{c}87.5 \% \\
(85.1-89.5 \%)\end{array}$ & B.1.1.7 \\
\hline & & $25,148 / 107,727$ & $\geq 16$ & BNT162b2 & $\begin{array}{c}\text { Symptomatic } \\
\text { infection of B.1.1.7 }\end{array}$ & $\geq 21$ & $\begin{array}{c}47.5 \% \\
(41.6-52.8 \%)\end{array}$ & $\geq 14$ & $\begin{array}{c}93.7 \% \\
(91.6-95.3 \%)\end{array}$ & B.1.1.7 \\
\hline & & $54,517 / 107,727$ & $\geq 16$ & ChAdOx1 & $\begin{array}{c}\text { Symptomatic } \\
\text { infection of B.1.1.7 }\end{array}$ & $\geq 21$ & $\begin{array}{c}48.7 \% \\
(45.2-51.9 \%)\end{array}$ & $\geq 14$ & $\begin{array}{c}74.5 \% \\
(68.4-79.4 \%)\end{array}$ & B.1.1.7 \\
\hline & & $79,665 / 107,727$ & $\geq 16$ & $\begin{array}{l}\text { BNT162b2, } \\
\text { ChAdOx1 }\end{array}$ & $\begin{array}{c}\text { Symptomatic } \\
\text { infection of B.1.617.2 }\end{array}$ & $\geq 21$ & $\begin{array}{c}30.7 \% \\
(25.2-35.7 \%)\end{array}$ & $\geq 14$ & $\begin{array}{c}79.6 \% \\
(76.7-82.1 \%)\end{array}$ & B.1.617.2 \\
\hline & & $54,517 / 107,727$ & $\geq 16$ & ChAdOx1 & $\begin{array}{c}\text { Symptomatic } \\
\text { infection of B.1.617.2 }\end{array}$ & $\geq 21$ & $\begin{array}{c}30.0 \% \\
(24.3-35.3 \%) \\
\end{array}$ & $\geq 14$ & $\begin{array}{c}67.0 \% \\
(61.3-71.8 \%) \\
\end{array}$ & B.1.617.2 \\
\hline \multirow[t]{2}{*}{$\begin{array}{l}\text { Vahidy et al./ } \\
\text { US [49] }^{\text {b }}\end{array}$} & \multirow[t]{2}{*}{ Cohort study } & $27,203 / 63,931$ & All & $\begin{array}{l}\text { BNT162b2, } \\
\text { mRNA-1273 }\end{array}$ & Hospitalization & $>14$ & $\begin{array}{c}77 \% \\
(71-82 \%)\end{array}$ & $>7$ & $\begin{array}{c}96 \% \\
(95-99 \%)\end{array}$ & $\begin{array}{c}\text { B.1, B.1.2, } \\
\text { B.1.596, } \\
\text { B.1.1.7 }\end{array}$ \\
\hline & & $27,203 / 63,931$ & All & $\begin{array}{l}\text { BNT162b2, } \\
\text { mRNA-1273 }\end{array}$ & Death & $>14$ & $\begin{array}{c}64.2 \% \\
(13.0-85.2 \%)\end{array}$ & $>7$ & $\begin{array}{c}98.7 \% \\
(91.0-99.8 \%)\end{array}$ & $\begin{array}{c}\text { B.1, B.1.2, } \\
\text { B.1.596, } \\
\text { B.1.1.7 }\end{array}$ \\
\hline \multirow[t]{2}{*}{$\begin{array}{l}\text { Baum et al.// } \\
\text { Finland [18] }^{\mathrm{b}}\end{array}$} & Cohort study & $758,437 / 95,719$ & $\geq 70$ & $\begin{array}{l}\text { BNT162b2, } \\
\text { mRNA-1273 }\end{array}$ & Overall infection & $21-27$ & $\begin{array}{c}41 \% \\
(25-54 \%)\end{array}$ & $\geq 7$ & $\begin{array}{c}75 \% \\
(65-82 \%)\end{array}$ & B.1.1.7 \\
\hline & Cohort study & $758,437 / 95,719$ & $\geq 70$ & $\begin{array}{l}\text { BNT162b2, } \\
\text { mRNA-1273 }\end{array}$ & Hospitalization & $21-27$ & $\begin{array}{c}57 \% \\
(24-75 \%)\end{array}$ & $\geq 7$ & $\begin{array}{c}93 \% \\
(70-98 \%)\end{array}$ & B.1.1.7 \\
\hline
\end{tabular}


Table 1. Cont.

\begin{tabular}{|c|c|c|c|c|c|c|c|c|c|c|}
\hline \multicolumn{11}{|c|}{ The Effectiveness of COVID-19 Vaccines among General Population } \\
\hline $\begin{array}{c}\text { First } \\
\text { Author/Country }\end{array}$ & Study Design & $\begin{array}{l}\text { No. of Vaccinated/No. } \\
\text { of Unvaccinated }\end{array}$ & Age (Years) & Vaccine & Outcomes & $\begin{array}{l}\text { Days after the } \\
\text { 1st Dose }\end{array}$ & $\begin{array}{l}\text { VE of 1st Dose } \\
\text { (95\% CI) }\end{array}$ & $\begin{array}{l}\text { Days after the } \\
\text { 2nd Dose }\end{array}$ & $\begin{array}{l}\text { VE of 2nd Dose } \\
\text { (95\% CI) }\end{array}$ & $\begin{array}{l}\text { Variants } \\
\text { Involved }\end{array}$ \\
\hline $\begin{array}{l}\text { Chodick et al./ } \\
\text { Israel [24] }\end{array}$ & Cohort study & $\begin{array}{c}503,875 \\
(351,897 \text { had follow-up } \\
\text { data for days } 13 \text { to } 24)\end{array}$ & $\geq 16$ & BNT162b2 & Overall infection & $13-24$ & $\begin{array}{c}51.4 \% \\
(16.3-71.8 \%)\end{array}$ & $\mathrm{N} / \mathrm{A}$ & $\mathrm{N} / \mathrm{A}$ & \\
\hline \multirow[t]{2}{*}{$\begin{array}{l}\text { Chodick et al./ } \\
\text { Israel [25] }\end{array}$} & Cohort study & $\begin{array}{c}1,178,597 \\
(872,454 \text { reach protection } \\
\text { period })\end{array}$ & $\geq 16$ & BNT162b2 & Overall infection & $\mathrm{N} / \mathrm{A}$ & $\mathrm{N} / \mathrm{A}$ & $7-27$ & $\begin{array}{c}90 \% \\
(79-95 \%)\end{array}$ & \\
\hline & & $\begin{array}{c}1,178,597 \\
\text { (872,454 reach protection } \\
\text { period) }\end{array}$ & $\geq 16$ & BNT162b2 & $\begin{array}{l}\text { Symptomatic } \\
\text { infection }\end{array}$ & $\mathrm{N} / \mathrm{A}$ & $\mathrm{N} / \mathrm{A}$ & $7-27$ & $\begin{array}{c}94 \% \\
(88-97 \%)\end{array}$ & \\
\hline \multirow[t]{9}{*}{$\begin{array}{l}\text { Flacco et al./ } \\
\text { Italy [31] }\end{array}$} & Cohort study & $69,539 / 175,687$ & $\geq 18$ & $\begin{array}{l}\text { BNT162b2, } \\
\text { ChAdOx1, } \\
\text { mRNA-1273 }\end{array}$ & Overall infection & & $\begin{array}{c}84 \% \\
(80-87 \%)\end{array}$ & $\geq 14$ & $\begin{array}{c}98 \% \\
(97-99 \%)\end{array}$ & B.1.1.7 \\
\hline & & $47,654 / 175,687$ & $\geq 18$ & BNT162b2 & Overall infection & $\geq 14$ & $\begin{array}{c}55 \% \\
(40-66 \%)\end{array}$ & $\geq 14$ & $\begin{array}{c}98 \% \\
(96-99 \%)\end{array}$ & B.1.1.7 \\
\hline & & $16,997 / 175,687$ & $\geq 18$ & ChAdOx1 & Overall infection & $\geq 21$ & $\begin{array}{c}95 \% \\
(92-97 \%)\end{array}$ & $\geq 14$ & $\mathrm{~N} / \mathrm{A}$ & B.1.1.7 \\
\hline & & $4888 / 175,687$ & $\geq 18$ & mRNA-1273 & Overall infection & $\geq 14$ & $\begin{array}{c}93 \% \\
(74-98 \%)\end{array}$ & $\geq 14$ & $100 \%$ & B.1.1.7 \\
\hline & & $47,654 / 175,687$ & $\geq 18$ & BNT162b2 & Hospitalization & $\geq 14$ & $\mathrm{~N} / \mathrm{A}$ & $\geq 14$ & $\begin{array}{c}99 \% \\
(96-100 \%)\end{array}$ & B.1.1.7 \\
\hline & & $\begin{array}{c}16,997 / 175,687 \\
4888 / 175,687\end{array}$ & $\begin{array}{l}\geq 18 \\
\geq 18\end{array}$ & $\begin{array}{l}\text { ChAdOx1 } \\
\text { mRNA-1273 }\end{array}$ & $\begin{array}{l}\text { Hospitalization } \\
\text { Hospitalization }\end{array}$ & $\begin{array}{l}\geq 21 \\
>14\end{array}$ & $\begin{array}{l}100 \% \\
\mathrm{~N} / \mathrm{A}\end{array}$ & $\begin{array}{l}\geq 14 \\
>14\end{array}$ & $\begin{array}{l}\mathrm{N} / \mathrm{A} \\
100 \%\end{array}$ & $\begin{array}{l}\text { B.1.1.7 } \\
\text { B.1.1.7 }\end{array}$ \\
\hline & & $69,539 / 175,687$ & $\geq 18$ & $\begin{array}{l}\text { BNT162b2, } \\
\text { ChAdOx1, } \\
\text { mRNA-1273 }\end{array}$ & Death & & $\begin{array}{c}73 \% \\
(-10-93 \%)\end{array}$ & $\geq 14$ & $\begin{array}{c}98 \% \\
(88-100 \%)\end{array}$ & B.1.1.7 \\
\hline & & $47,654 / 175,687$ & $\geq 18$ & BNT162b2 & Death & $\geq 14$ & N/A & $\geq 14$ & $\begin{array}{c}98 \% \\
(87-100 \%)\end{array}$ & B.1.1.7 \\
\hline & & $\begin{array}{c}16,997 / 175,687 \\
4888 / 175,687\end{array}$ & $\begin{array}{l}\geq 18 \\
\geq 18\end{array}$ & $\begin{array}{l}\text { ChAdOx1 } \\
\text { mRNA-1273 }\end{array}$ & $\begin{array}{l}\text { Death } \\
\text { Death }\end{array}$ & $\begin{array}{l}\geq 21 \\
\geq 14\end{array}$ & $\begin{array}{l}100 \% \\
\mathrm{~N} / \mathrm{A}\end{array}$ & $\begin{array}{l}\geq 14 \\
\geq 14\end{array}$ & $\begin{array}{l}\mathrm{N} / \mathrm{A} \\
100 \%\end{array}$ & $\begin{array}{l}\text { B.1.1.7 } \\
\text { B.1.1.7 }\end{array}$ \\
\hline
\end{tabular}


Table 1. Cont.

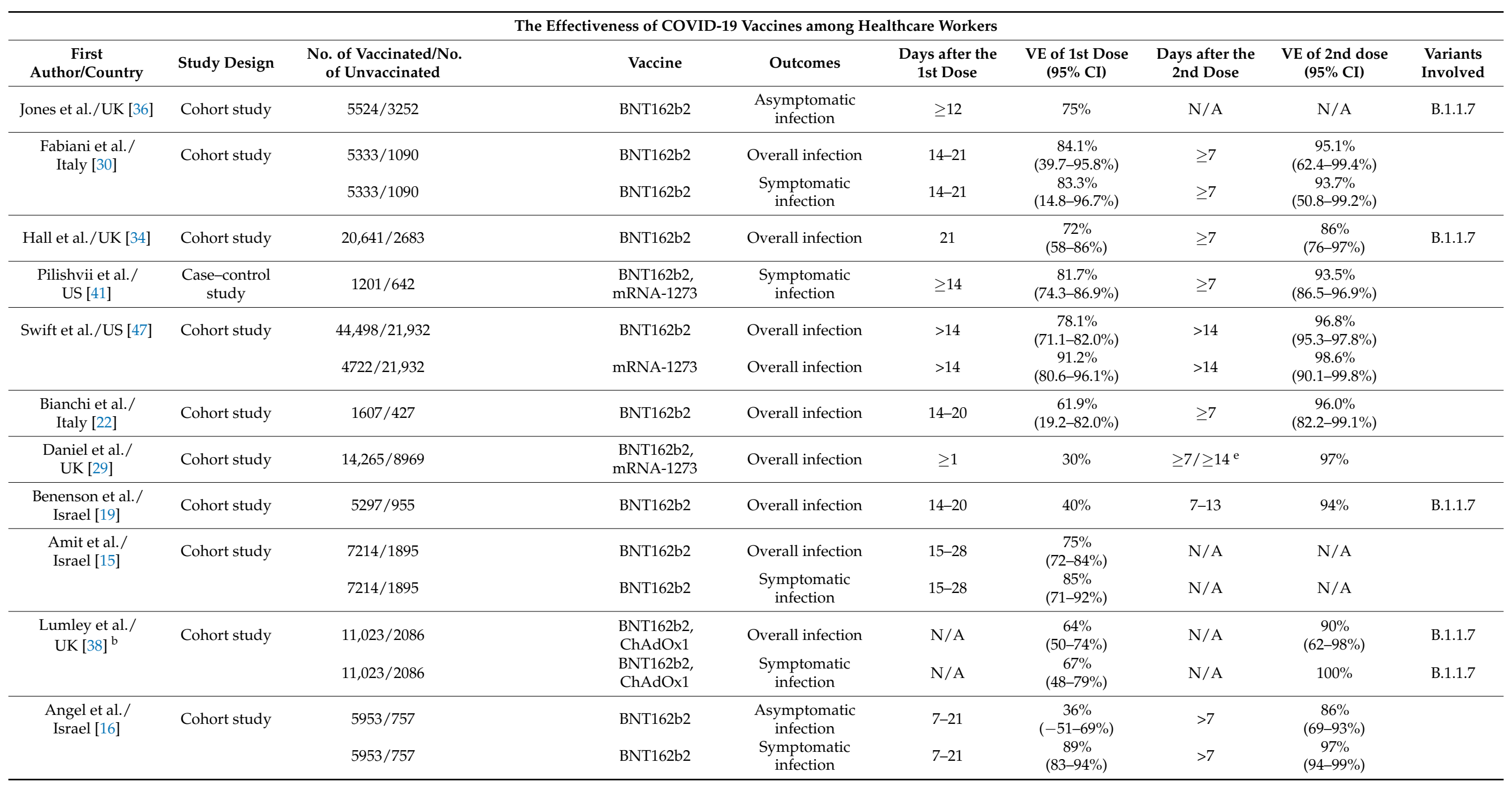


Table 1. Cont.

\begin{tabular}{|c|c|c|c|c|c|c|c|c|c|c|}
\hline \multicolumn{11}{|c|}{ The Effectiveness of COVID-19 Vaccines among Healthcare Workers } \\
\hline $\begin{array}{c}\text { First } \\
\text { Author/Country }\end{array}$ & Study Design & $\begin{array}{l}\text { No. of Vaccinated/No. } \\
\text { of Unvaccinated }\end{array}$ & & Vaccine & Outcomes & $\begin{array}{l}\text { Days after the } \\
\text { 1st Dose }\end{array}$ & $\begin{array}{l}\text { VE of 1st Dose } \\
(95 \% \mathrm{CI})\end{array}$ & $\begin{array}{l}\text { Days after the } \\
\text { 2nd Dose }\end{array}$ & $\begin{array}{l}\text { VE of 2nd dose } \\
(95 \% \mathrm{CI})\end{array}$ & $\begin{array}{l}\text { Variants } \\
\text { Involved }\end{array}$ \\
\hline $\begin{array}{l}\text { Moustsen-Helms } \\
\text { et al./ } \\
\text { Denmark [39] }^{\text {a }}\end{array}$ & Cohort study & $91,865 / 239,174$ & & BNT162b2 & Overall infection & $>14$ & $\begin{array}{c}17 \% \\
(4-28 \%)\end{array}$ & $>7$ & $\begin{array}{c}90 \% \\
(82-95 \%)\end{array}$ & \\
\hline $\begin{array}{l}\text { Emborg et al./ } \\
\text { Denmark [11] }{ }^{\mathrm{b}}\end{array}$ & Cohort study & $119,951 / 305,848$ & & BNT162b2 & Overall infection & $\mathrm{N} / \mathrm{A}$ & $\mathrm{N} / \mathrm{A}$ & 7 & $\begin{array}{c}80 \% \\
(77-83 \%)\end{array}$ & \\
\hline $\begin{array}{l}\text { Azamgarhi et al./ } \\
\text { UK [17] }\end{array}$ & Cohort study & $1409 / 851$ & & BNT162b2 & Overall infection & $\geq 14$ & $\begin{array}{c}70 \% \\
(6-91 \%)\end{array}$ & $\mathrm{N} / \mathrm{A}$ & $\mathrm{N} / \mathrm{A}$ & \\
\hline \multirow[t]{3}{*}{$\begin{array}{c}\text { Thompson et al./ } \\
\text { US [48] }\end{array}$} & Cohort study & $3179 / 796$ & & $\begin{array}{l}\text { BNT162b2, } \\
\text { mRNA-1273 }\end{array}$ & Overall infection & $\geq 14$ & $\begin{array}{c}81 \% \\
(64-90 \%)\end{array}$ & $\geq 14$ & $\begin{array}{c}91 \% \\
(76-97 \%)\end{array}$ & $\begin{array}{c}\text { B.1.429, } \\
\text { B.1.427, } \\
\text { B.1.1.7, P.2 }\end{array}$ \\
\hline & & & & BNT162b2 & Overall infection & $\geq 14$ & $\begin{array}{c}80 \% \\
(60-90 \%)\end{array}$ & $\geq 14$ & $\begin{array}{c}93 \% \\
(78-98 \%)\end{array}$ & $\begin{array}{c}\text { B.1.429, } \\
\text { B.1.427, } \\
\text { B.1.1.7, P.2 }\end{array}$ \\
\hline & & & & mRNA-1273 & Overall infection & $\geq 14$ & $\begin{array}{c}83 \% \\
(40-95 \%)\end{array}$ & $\geq 14$ & $\begin{array}{c}82 \% \\
(20-96 \%)\end{array}$ & $\begin{array}{c}\text { B.1.429, } \\
\text { B.1.427, } \\
\text { B.1.1.7, P.2 }\end{array}$ \\
\hline $\begin{array}{l}\text { Hitchings et al./ } \\
\text { Brazil [35] }^{\mathrm{b}}\end{array}$ & $\begin{array}{l}\text { Case-control } \\
\text { study }\end{array}$ & $47,170 / 5983$ & & CoronaVac & $\begin{array}{c}\text { Symptomatic } \\
\text { infection }\end{array}$ & $\mathrm{N} / \mathrm{A}$ & N/A & $\geq 14$ & $\begin{array}{c}36.8 \% \\
(54.9-74.2 \%) \\
\end{array}$ & P.1 \\
\hline $\begin{array}{l}\text { Shrestha et al./ } \\
\text { US [44] }^{\text {b }}\end{array}$ & Cohort study & $28,223 / 18,643$ & & $\begin{array}{l}\text { BNT162b2, } \\
\text { mRNA-1273 }\end{array}$ & Overall infection & 14 & $\begin{array}{c}95.0 \% \\
(93.0-96.4 \%)\end{array}$ & $\geq 14$ & $\begin{array}{c}97.1 \% \\
(94.3-98.5 \%)\end{array}$ & \\
\hline \multicolumn{11}{|c|}{ The Effectiveness of COVID-19 Vaccines among Residents of a Long-Term Care Facility, Subjects with Comorbidity, Subjects with Chronic Illness, or Elderly People ( $\geq 65$ Years) Requiring Personal Care } \\
\hline $\begin{array}{c}\text { First } \\
\text { Author/Country }\end{array}$ & Study Design & $\begin{array}{l}\text { No. of Vaccinated/No. } \\
\text { of Unvaccinated }\end{array}$ & Participants & Vaccine & Outcomes & $\begin{array}{l}\text { Days after the } \\
\text { 1st Dose }\end{array}$ & $\begin{array}{l}\text { VE of 1st Dose } \\
\quad(95 \% \mathrm{CI})\end{array}$ & $\begin{array}{l}\text { Days after the } \\
\text { 2nd dose }\end{array}$ & $\begin{array}{l}\text { VE of 2nd Dose } \\
(95 \% \mathrm{CI})\end{array}$ & $\begin{array}{l}\text { Variants } \\
\text { Involved }\end{array}$ \\
\hline $\begin{array}{l}\text { Moustsen-Helms } \\
\text { et al./ } \\
\text { Denmark [39] }^{\text {a }}\end{array}$ & Cohort study & $37,172 / 1868$ & RLCF & BNT162b2 & Overall infection & $>14$ & $\begin{array}{c}21 \% \\
(-11-44 \%)\end{array}$ & $>7$ & $\begin{array}{c}64 \% \\
(14-84 \%)\end{array}$ & \\
\hline \multirow[t]{3}{*}{$\begin{array}{l}\text { Shrotri et al./ } \\
\text { UK [45] }\end{array}$} & Cohort study & $9160 / 1252$ & RLCF & $\begin{array}{l}\text { BNT162b2, } \\
\text { ChAdOx1 }\end{array}$ & Overall infection & $35-48$ & $\begin{array}{c}62 \% \\
(23-81 \%)\end{array}$ & $\mathrm{N} / \mathrm{A}$ & N/A & \\
\hline & & $3022 / 1252$ & RLCF & BNT162b2 & Overall infection & $35-48$ & $\begin{array}{c}65 \% \\
(29-83 \%)\end{array}$ & $\mathrm{N} / \mathrm{A}$ & $\mathrm{N} / \mathrm{A}$ & \\
\hline & & $6138 / 1252$ & RLCF & ChAdOx1 & Overall infection & $35-48$ & $\begin{array}{c}68 \% \\
(34-85 \%)\end{array}$ & N/A & N/A & \\
\hline
\end{tabular}


Table 1. Cont

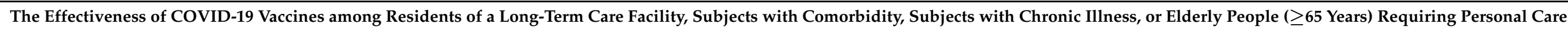

\begin{tabular}{|c|c|c|c|c|c|c|c|c|c|c|}
\hline $\begin{array}{c}\text { First } \\
\text { Author/Country }\end{array}$ & Study Design & $\begin{array}{l}\text { No. of Vaccinated/No. } \\
\text { of Unvaccinated }\end{array}$ & Participants & Vaccine & Outcomes & $\begin{array}{l}\text { Days after the } \\
\text { 1st Dose }\end{array}$ & $\begin{array}{l}\text { VE of 1st Dose } \\
\text { (95\% CI) }\end{array}$ & $\begin{array}{l}\text { Days after the } \\
\text { 2nd dose }\end{array}$ & $\begin{array}{l}\text { VE of 2nd Dose } \\
(95 \% \mathrm{CI})\end{array}$ & $\begin{array}{l}\text { Variants } \\
\text { Involved }\end{array}$ \\
\hline \multirow[t]{8}{*}{$\begin{array}{l}\text { Emborg et al./ } \\
\text { Denmark [11] }\end{array}$} & \multirow[t]{8}{*}{ Cohort study } & $42744 / 3357$ & RLCF & BNT162b2 & Overall infection & N/A & N/A & 7 & $\begin{array}{c}53 \% \\
(29-69 \%)\end{array}$ & \\
\hline & & $51,311 / 10,494$ & $65 \mathrm{PHC}$ & BNT162b2 & Overall infection & N/A & N/A & 7 & $\begin{array}{c}86 \% \\
(78-91 \%)\end{array}$ & \\
\hline & & $180,766 / 51,092$ & SCC & BNT162b2 & Overall infection & $\mathrm{N} / \mathrm{A}$ & $\mathrm{N} / \mathrm{A}$ & 7 & $\begin{array}{c}71 \% \\
(58-80 \%)\end{array}$ & \\
\hline & & $42,744 / 3357$ & RLCF & BNT162b2 & Hospitalization & N/A & N/A & 7 & $\begin{array}{c}75 \% \\
(49-89 \%)\end{array}$ & \\
\hline & & $51,311 / 10,494$ & $65 \mathrm{PHC}$ & BNT162b2 & Hospitalization & N/A & N/A & 7 & $\begin{array}{c}87 \% \\
(70-95 \%)\end{array}$ & \\
\hline & & $180,766 / 51,092$ & SCC & BNT162b2 & Hospitalization & N/A & N/A & 7 & $\begin{array}{c}81 \% \\
(49-93 \%)\end{array}$ & \\
\hline & & $42,744 / 3357$ & RLCF & BNT162b2 & Death & N/A & N/A & 7 & $\begin{array}{c}89 \% \\
(81-93 \%)\end{array}$ & \\
\hline & & $51,311 / 10,494$ & $65 \mathrm{PHC}$ & BNT162b2 & Death & N/A & N/A & 7 & $\begin{array}{c}97 \% \\
(88-99 \%)\end{array}$ & \\
\hline \multirow[t]{3}{*}{$\begin{array}{l}\text { Mazagatos et al./ } \\
\text { Spain [12] }\end{array}$} & \multirow[t]{3}{*}{$\begin{array}{l}\text { Case-control } \\
\text { study }\end{array}$} & $300,133 / 38,012$ & $\begin{array}{c}\text { RLCF } \\
(\geq 65 \mathrm{y} / \mathrm{o})\end{array}$ & $\begin{array}{l}\text { BNT162b2, } \\
\text { mRNA-1273 }\end{array}$ & Overall infection & $>14$ & $\begin{array}{c}50.5 \% \\
(37.1-61.1 \%)\end{array}$ & $\geq 7 / \geq 14^{\mathrm{e}}$ & $\begin{array}{c}71.4 \% \\
(55.7-81.5 \%)\end{array}$ & \\
\hline & & $300,133 / 38,012$ & $\begin{array}{c}\text { RLCF } \\
(\geq 65 \mathrm{y} / \mathrm{o})\end{array}$ & $\begin{array}{l}\text { BNT162b2, } \\
\text { mRNA-1273 }\end{array}$ & $\begin{array}{l}\text { Asymptomatic } \\
\text { infection }\end{array}$ & $>14$ & $\begin{array}{c}58.0 \% \\
(41.7-69.7 \%)\end{array}$ & $\geq 7 / \geq 14^{\mathrm{e}}$ & $\begin{array}{c}69.7 \% \\
(47.7-82.5 \%)\end{array}$ & \\
\hline & & $300,133 / 38,012$ & $\begin{array}{c}\text { RLCF } \\
(\geq 65 \mathrm{y} / \mathrm{o})\end{array}$ & $\begin{array}{l}\text { BNT162b2, } \\
\text { mRNA-1273 }\end{array}$ & Death & $>14$ & $\begin{array}{c}55.6 \% \\
(26.6-73.2 \%)\end{array}$ & $\geq 7 / \geq 14^{\mathrm{e}}$ & $\begin{array}{c}97.0 \% \\
(91.7-98.9 \%)\end{array}$ & \\
\hline
\end{tabular}

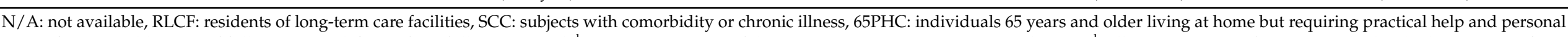

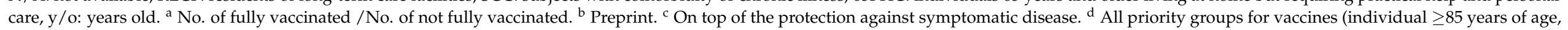

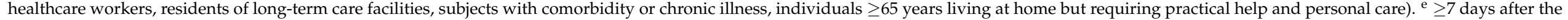
second dose for BNT162b2 and $>14$ days for mRNA-1273. 


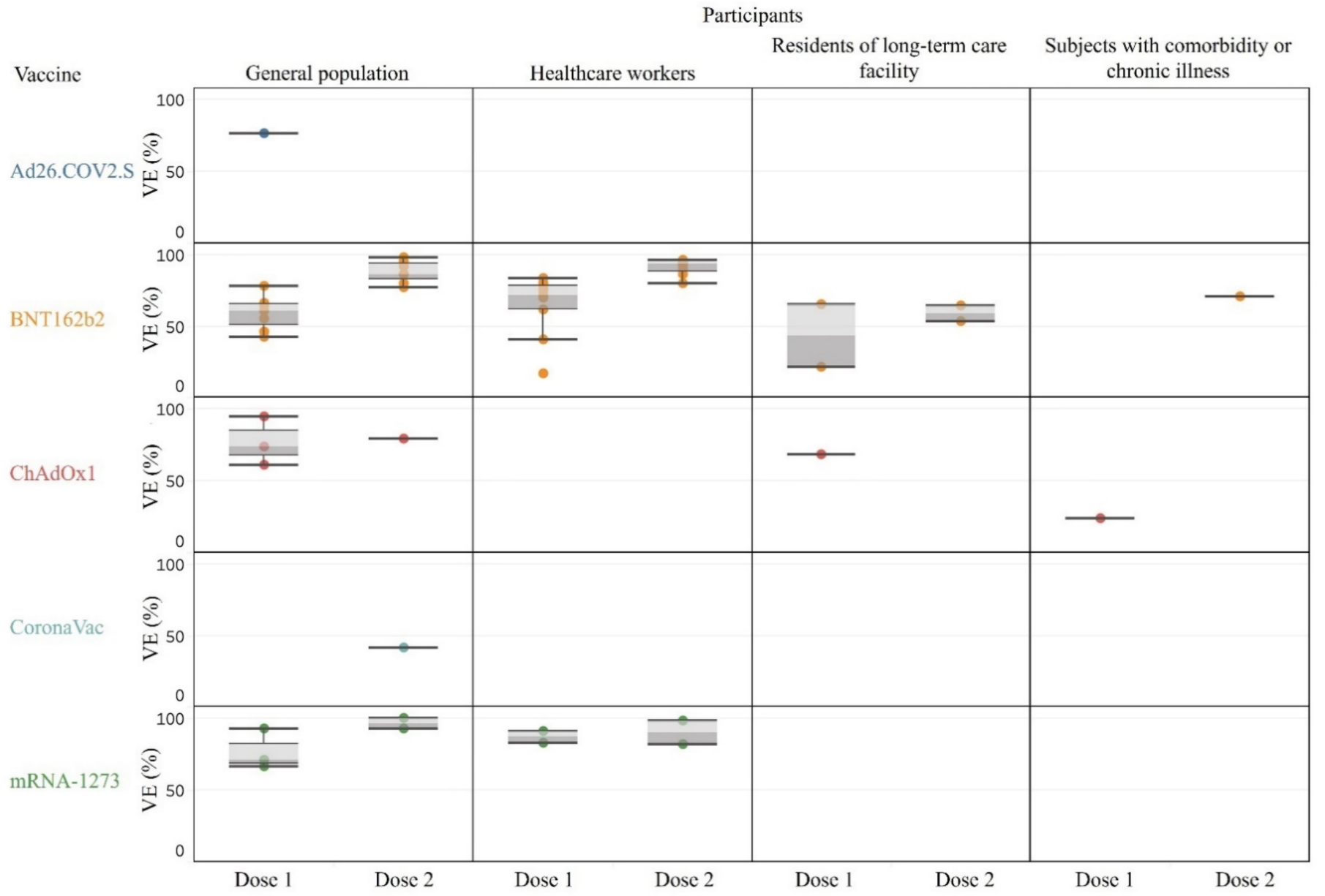

Figure 2. Vaccine effectiveness against overall SARS-CoV-2 infection.

3.2.2. Asymptomatic Infection, Symptomatic Infection, Hospitalization, Critical Disease, and Death

The VE estimates against SARS-CoV-2-related various outcomes are shown in Table 1, Figure 3A-D, and Supplementary Figure S1. Among the general population in the UK, the VE against asymptomatic infection after full vaccination either with BNT162b2 (VE: 58\%) or ChAdOx1 (VE: 61\%) was not as high as that in Israel (VE: 92\%) (Figure 3A, Table 1A) [33,42]. Much more beneficial effects were found when symptomatic infection reduction was considered as an outcome, with the VE reaching up to $89-97 \%$ for BNT162b2 [26,28,33,37,42], 92\% for ChAdOx1 [42], and 94\% for mRNA-1273 after full vaccination (Table 1, Figure 3B) [26]. Studies in healthcare workers also showed beneficial effects from vaccination (VE: 94-97\% by two doses of BNT162b2) [15,16,30], except for a CoronaVac study in Brazil, which showed a low VE against symptomatic infection with the predominant P.1 variant (37\%, $95 \%$ CI, 54.9-74.2\%) even after being fully vaccinated (Figure 3B, Table 1) [35]. The protective effects become more obvious for more severe outcomes. The VE against critical disease was higher than $90 \%$ by BNT162b2 [28,33,40]. A study even demonstrated a VE of $100 \%$ for subjects who were fully vaccinated with mRNA-1273 (see Supplementary Figure S1) [40]. 

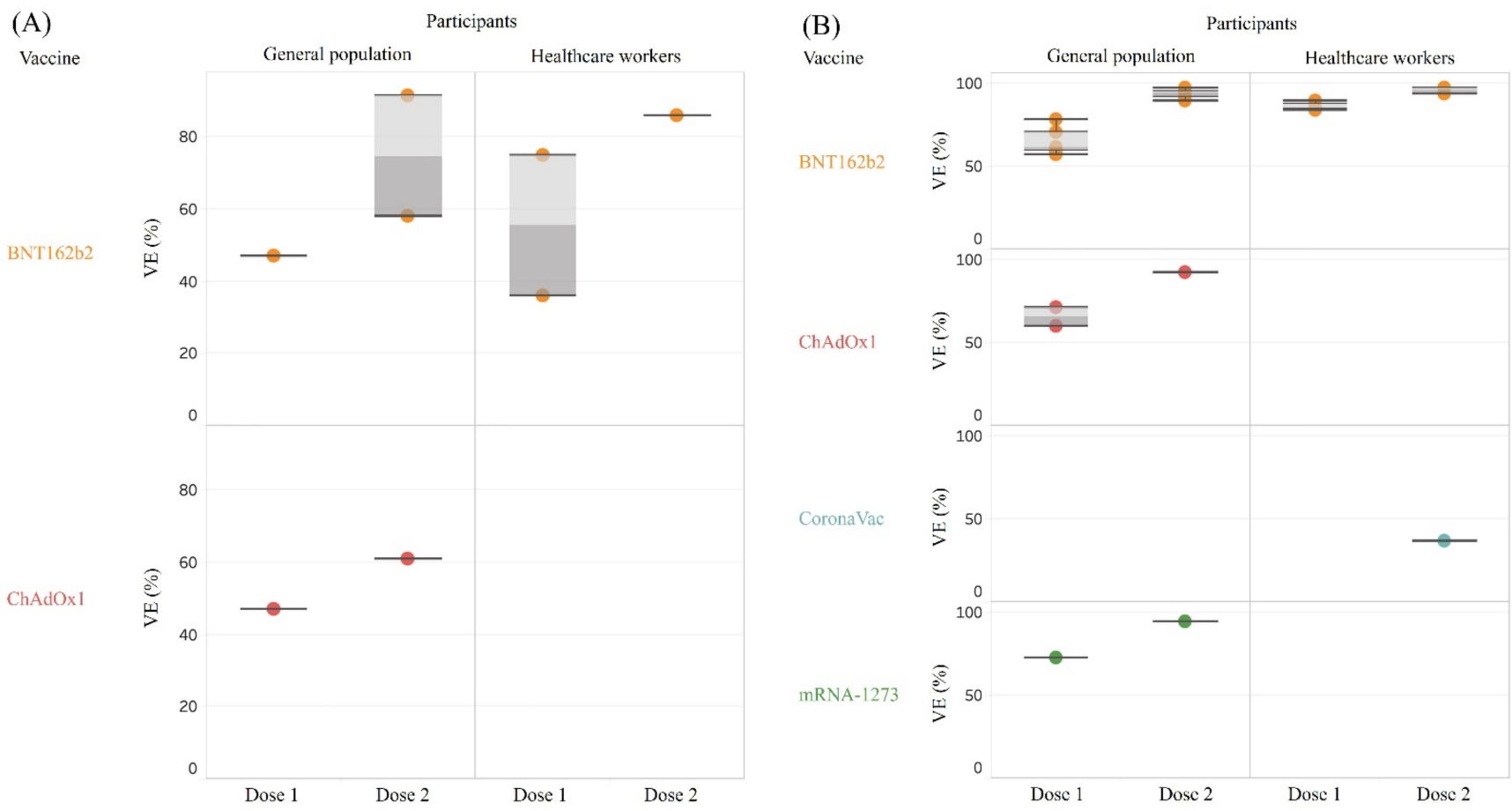

(C)

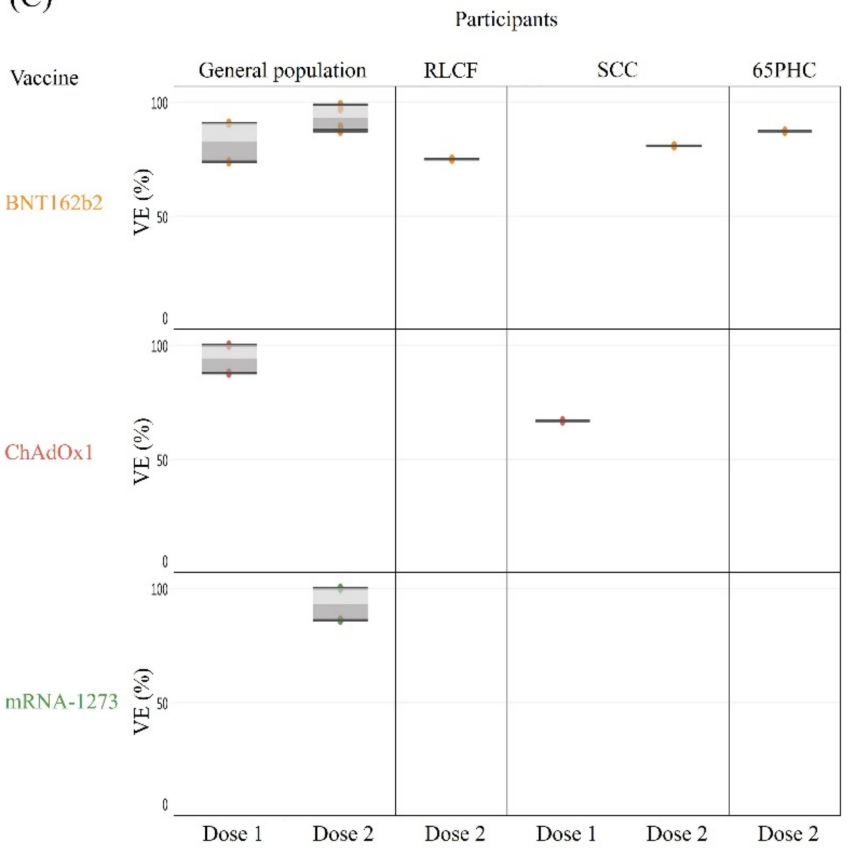

(D)

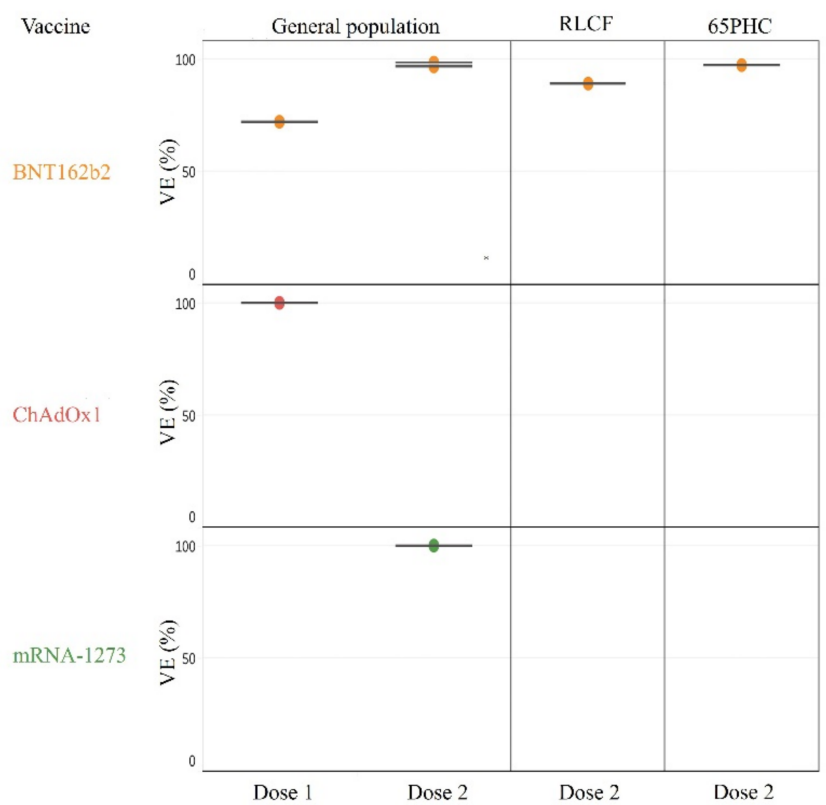

Figure 3. Vaccine effectiveness against various outcomes. (A) Vaccine effectiveness against asymptomatic infection. (B) Vaccine effectiveness against symptomatic infection. (C) Vaccine effectiveness against hospitalization. (D) Vaccine effectiveness against death. Abbreviations: RLCF: residents of long-term care facilities, SCC: subjects with comorbidity or chronic illness, 65PHC: individuals 65 years and older living at home but requiring practical help and personal care.

Regarding the risk reduction on hospitalization among the general population, the effectiveness was similar among one dose of ChAdOx1 (VE: 88-100\%) [31,50], two doses of BNT162b2 (VE: 87-99\%) [11,28,33,50], and two doses of mRNA-1273 (VE: 86-100\%) (Figure 3C) [31,40]. Reduction of death for more than $95 \%$ was also found by one dose of ChAdOx1 and two doses of mRNA vaccines (Figure 3D). The VE estimates against COVID-19 hospital admissions after vaccination either with BNT162b2 or ChAdOx1 in 
older age groups were slightly lower than that of younger age groups [50]. The result of VE across age groups is shown in Supplementary Table S3.

\subsection{Effectiveness of Vaccines against SARS-CoV-2 VOC}

Three studies specifically calculated the VE against the VOC $[9,20,46]$ (Table 1, Figure 4). For the Alpha variant (B.1.1.7), one dose of BNT162b2 or ChAdOx1 provided around 50\% of VE on symptomatic infection and critical disease or death [9,32]. For the delta variant (B.1.617.2), better VE was also found for two doses of BNT162b2 (VE: 93.4\%, 95\% CI, 90.4$95.5 \%)$ than in ChAdOx1 (66.1\%, 95\% CI, 54.0-75.0\%) in terms of preventing symptomatic infection [32].

\section{Outcomes}

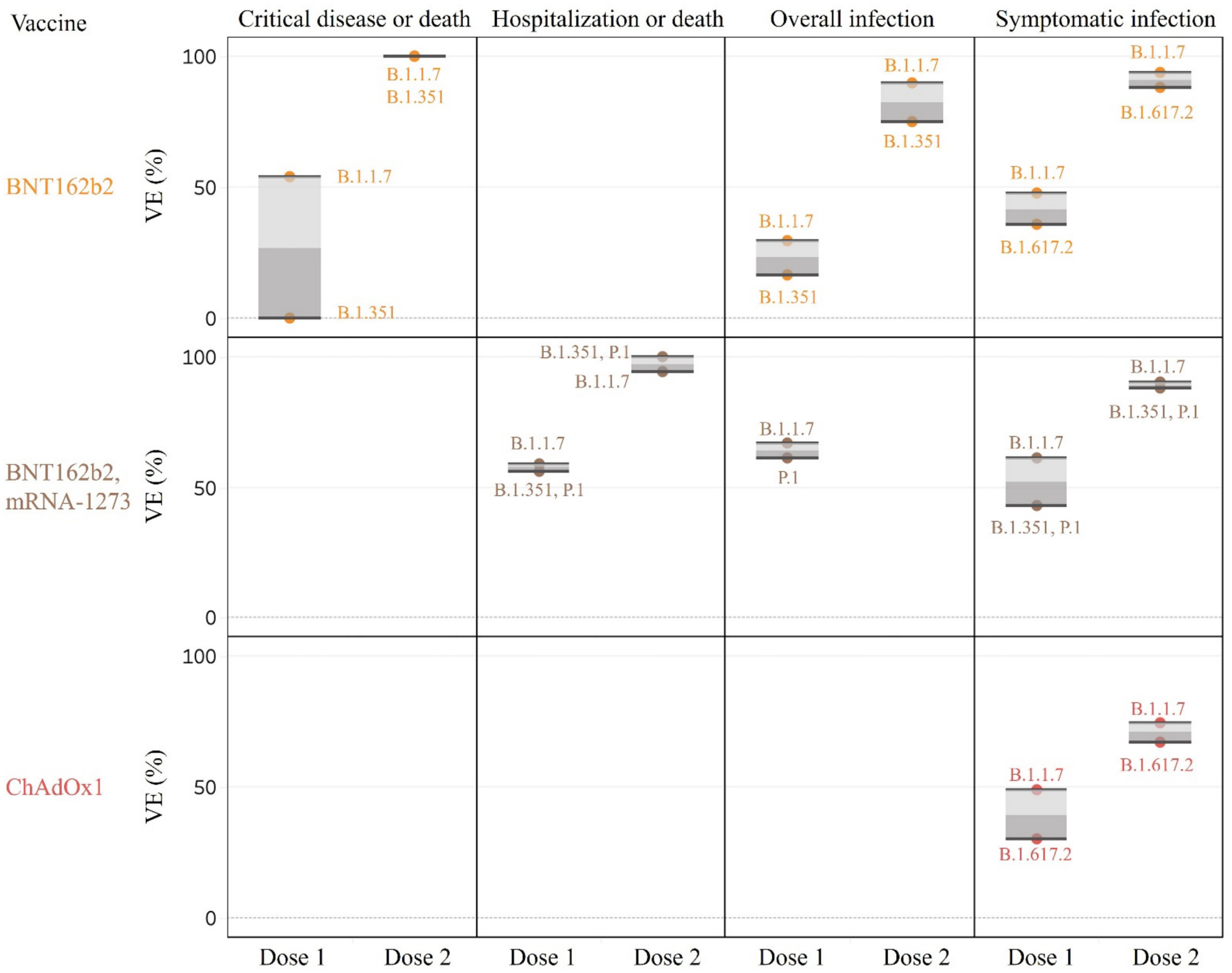

Figure 4. Vaccine effectiveness against SARS-CoV-2 variants of concern.

\section{Discussion}

This rapid review included 39 studies from 11 countries. Some of these studies may have overlapping populations with data obtained from the same datasets (e.g., national registry) or from the same institution or same region [15-17,19-21,28,32,34,36-39,41,42,45]. By excluding possible double counted participants, this review approximately covered a population of 15 million in total with over 8 million vaccinated subjects. The results of all included studies consistently show that vaccination among the general population 
in real-world settings, with either BNT162b2, ChAdOx1, or mRNA-1273, substantially reduced the risk of overall SARS-CoV-2 infection. Though the effectiveness of the vaccine in residents of long-term care facilities is lower than that in the general population, studies showed that just one dose of the BNT162b2 or ChAdOx1 vaccine can still provide a VE of $60 \%$ or higher. Their protective effectiveness became more obvious as the severity of the clinical endpoint increased. The effectiveness of one dose of ChAdOx1 in preventing hospitalization and death was higher than $80 \%$ in the general population and similar to that observed in subjects fully vaccinated with BNT162b2 or mRNA-1273. While the delta variant is becoming the dominant strain circulating in many parts of the world, VE reports on the delta variant are scarce. One recently published study shows that two doses of BNT162b2 can significantly reduce the risk of delta variant-related symptomatic infections [20].

The suboptimal VE observed in the residents of long-term care facilities is probably due to immunosenescence, which leads to defects in innate and adaptive immune responses. Vaccine responses tended to be weaker and declined earlier [51] with a lower titer of antibodies [52-54]. Since they are vulnerable to developing SARS-CoV-2 infections and its complications, improved vaccine strategies or further vaccine boosting are required in these vulnerable populations.

It should be noted that VE between vaccines cannot be directly compared across these papers due to the following reasons. The first one is the heterogeneity in the interval between vaccination and the start of event measurement. The VE of the first dose of BNT162b2 against infection was $61 \% \geq 14$ days after the vaccination in the study by Pawlowski et al. [40] and $72 \% \geq 21$ days after vaccination in the study by Hall et al. [34]. As immunity is gradually building [3], the protective effects would increase over a given period $[18,28]$. At present, there is no data to suggest the timing of the VE plateau for each vaccine. Second, large variation was noted regarding the dosing intervals. To vaccinate as many people as possible, the UK government decided that the dosing interval for vaccines (whether BNT162b2 or ChAdOx1) could be extended up to 12 weeks [21,37]. In Canada, the administration of the second dose of BNT162b2 and mRNA-1273 was delayed by up to 16 weeks for most individuals due to the disruption of the vaccine supply [26]. There is limited data about the change of the effectiveness for extended dosing intervals. Variations in dosing intervals would be very common in most countries of the world due to the insufficient and unstable vaccine supply. Evidence regarding the effectiveness with appropriate and tolerable dosing intervals are needed [55,56]. Third, some studies [12,29,38,41,44,49] estimated the VE by pooled analyses of two vaccines, in which we cannot obtain the VE of each type of vaccine. Fourth, population bias with unmatched control arms or unequal use of vaccine types across study cohorts could make the data of direct comparison of vaccine types unreliable [20,32,37,40,50]. Different vaccines may target different populations in the roll-out period. For example, old age and healthcare workers are in the priority group of vaccination programs in most countries, but the timescale of supply was different between vaccine types [18,32]. Although the demographic factors were controlled in most studies, residual confounding may not be entirely excluded.

There are several barriers and limitations in this study. First, the literature about the VE against COVID-19 is rapidly evolving and growing exponentially. In this review, 15 of the 39 included studies were preprint articles up to 30 June 2021. While we were processing the review and extracting the data, in 5 July studies were accepted with their data changed in an online version $[20,32,35,38,46,57]$ so that we need to constantly update the data accordingly before submission. Second, these included studies were disproportionately from countries in Europe, North America, and Israel, and were mostly focused on BNT162b2, because these countries had higher priority and access to a large amount of COVID-19 vaccines than most countries in other regions in the first half of 2021 [7]. In addition, shorter dosing intervals and a larger vaccine supply in these nations also make the data of VE associated with BNT162b2 much more comprehensive than other vaccines. Longer dosing intervals 
made the full vaccination reports of ChAdOx1 much fewer in number than those on mRNA vaccines among the included articles over the past months. Furthermore, the VE assessment reports on CoronaVac and Ad26.COV2.S are also too few to draw conclusions. Third, the VE estimates of all the studies were variable and mostly based on a relatively short study time (range, $<2$ months to 6 months) with a median follow-up period ranging from 28 to 106 days $[15,16,22,33,34,39,40,47]$. Further studies are needed to clarify the duration of the protective effects of vaccines. Fourth, studies focused on the VE against the variants of concern, in particularly the delta variant, which evolved as the dominant variant of SARS-CoV-2 in the first half-year of 2021, are scarce [32]. Additionally, the prevalence of the major variants is believed to be quite different among countries and subject to change over time, while the effectiveness of vaccines during the study time might be highly influenced by the local prevalence of the variants [43]. The heterogeneity across studies in the dosing interval, timing of outcome measurement, different target population between vaccine types, variations and shortness of study follow-up period, and scarce data about the prevalence of and the VE against major variants of SARS-CoV-2 all make meta-analyses for studies before 30 June 2021 extremely difficult and may possibly lead to biased results.

\section{Conclusions}

This rapid review provided timely and comprehensive evidence on the effectiveness of the ChAdOx1, BNT162b2, and mRNA-1273 vaccines against various COVID-19 infectionrelated endpoints ranging from asymptomatic to critical illness and death. This review highlights the VE across different segments of populations. These real-world results tend to support the implementation of mass vaccination campaigns as public health strategies, and may also help ease skepticism about VE, which is still a common problem in many parts of the world. However, data based on the studies of the first half-year of vaccine administration are not enough and may limit the conclusions regarding the mRNA vaccines and ChAdOx1. Further studies are needed to provide the information on different races/ethnicity, the effects against SARS-CoV-2 variants, and the duration of protection with longer study times.

Supplementary Materials: The following are available online at https:/ /www.mdpi.com/article/ 10.3390/vaccines9121489/s1, Figure S1: Vaccine effectiveness against critical disease, Table S1: Characteristics of the included studies, Table S2: Risk of bias assessment by ROBINS-I, Table S3: Summary of the studies on the effectiveness of COVID-19 vaccines across age groups.

Author Contributions: Conceptualization, C.-J.C., Y.S., and C.-Y.L. (Chung-Yi Li); methodology, C.-J.C. and Y.S.; systematic review, C.-J.C. and Y.S.; writing-original draft preparation, C.-J.C. and Y.S.; writing-review and editing, C.-Y.L. (Chun-Yi Lu), H.-J.C., C.-Y.L. (Chun-Yu Lee), C.-H.L. (Chang-Hsiu Liu), C.-H.L. (Cheng-Huai Lin), C.-J.L., and C.-Y.L. (Chung-Yi Li); visualization, C.-J.C. and Y.-H.C. All authors have read and agreed to the published version of the manuscript.

Funding: This research received no external funding.

Institutional Review Board Statement: Not applicable.

Informed Consent Statement: Not applicable.

Conflicts of Interest: The authors declare no conflict of interest.

\section{References}

1. Voysey, M.; Clemens, S.A.C.; Madhi, S.A.; Weckx, L.Y.; Folegatti, P.M.; Aley, P.K.; Angus, B.; Baillie, V.L.; Barnabas, S.L.; Bhorat, Q.E.; et al. Safety and efficacy of the chadox1 nCoV-19 vaccine (azd1222) against SARS-CoV-2: An interim analysis of four randomised controlled trials in Brazil, South Africa, and the UK. Lancet 2021, 397, 99-111. [CrossRef]

2. $\quad$ Baden, L.R.; El Sahly, H.M.; Essink, B.; Kotloff, K.; Frey, S.; Novak, R.; Diemert, D.; Spector, S.A.; Rouphael, N.; Creech, C.B.; et al. Efficacy and safety of the mRNA-1273 SARS-CoV-2 vaccine. N. Engl. J. Med. 2021, 384, 403-416. [CrossRef] [PubMed]

3. Polack, F.P.; Thomas, S.J.; Kitchin, N.; Absalon, J.; Gurtman, A.; Lockhart, S.; Perez, J.L.; Pérez Marc, G.; Moreira, E.D.; Zerbini, C.; et al. Safety and efficacy of the BNT162b2 mRNA COVID-19 vaccine. N. Engl. J. Med. 2020, 383, 2603-2615. [CrossRef] 
4. Al Kaabi, N.; Zhang, Y.; Xia, S.; Yang, Y.; Al Qahtani, M.M.; Abdulrazzaq, N.; Al Nusair, M.; Hassany, M.; Jawad, J.S.; Abdalla, J.; et al. Effect of 2 inactivated SARS-CoV-2 vaccines on symptomatic COVID-19 infection in adults: A randomized clinical trial. JAMA 2021, 326, 35-45. [CrossRef] [PubMed]

5. Tanriover, M.D.; Doğanay, H.L.; Akova, M.; Güner, H.R.; Azap, A.; Akhan, S.; Köse, Ş.; Erdinç, F.; Akalın, E.H.; Tabak, Ö.F.; et al. Efficacy and safety of an inactivated whole-virion SARS-CoV-2 vaccine (coronavac): Interim results of a double-blind, randomised, placebo-controlled, phase 3 trial in turkey. Lancet 2021, 398, 213-222. [CrossRef]

6. Sadoff, J.; Gray, G.; Vandebosch, A.; Cárdenas, V.; Shukarev, G.; Grinsztejn, B.; Goepfert, P.A.; Truyers, C.; Fennema, H.; Spiessens, B.; et al. Safety and efficacy of single-dose Ad26.COV2.S vaccine against COVID-19. N. Engl. J. Med. 2021, 384, $2187-2201$. [CrossRef]

7. Ledford, H. Six months of COVID vaccines: What 1.7 billion doses have taught scientists. Nature 2021, 594, 164-167. [CrossRef]

8. de Faria, E.; Guedes, A.R.; Oliveira, M.S.; de Godoy Moreira, M.V.; Maia, F.L.; dos Santos Barboza, A.; Leme, M.D.; Letaif, L.S.H.; Miethke-Morais, A.; Bonfá, E.; et al. Performance of vaccination with coronavac in a cohort of healthcare workers (hcw)-Preliminary report. medRxiv 2021, in press. [CrossRef]

9. Abu-Raddad, L.J.; Chemaitelly, H.; Butt, A.A. Effectiveness of the BNT162b2 COVID-19 Vaccine against the B.1.1.7 and B.1.351 Variants. N. Engl. J. Med. 2021, 385, 187-189. [CrossRef]

10. World Health Organization. Coronavirus Disease (COVID-19): Vaccines. Is There a Vaccine for COVID-19? Available online: https:/ / www.Who.Int/news-room/q-a-detail/coronavirus-disease-(COVID-19)-vaccines?Topicsurvey=)\&gclid=cj0kcqjw_ dwgbhdaarisamcyujwfzbr4gumousyvkz-qfk6khe6wywytf4rhhrkidoa3q7ja0gayl8oaaqtoealw_wcb (accessed on 22 June 2021).

11. Emborg, H.-D.; Valentiner-Branth, P.; Schelde, A.B.; Nielsen, K.F.; Gram, M.A.; Moustsen-Helms, I.R.; Chaine, M.; Seidelin, U.H.; Nielsen, J. Vaccine effectiveness of the BNT162b2 mRNA COVID-19 vaccine against rt-pcr confirmed SARS-CoV-2 infections, hospitalisations and mortality in prioritised risk groups. medRxiv 2021, in press. [CrossRef]

12. Mazagatos, C.; Monge, S.; Olmedo, C.; Vega, L.; Gallego, P.; Martín-Merino, E.; Sierra, M.J.; Limia, A.; Larrauri, A. Effectiveness of mRNA COVID-19 vaccines in preventing SARS-CoV-2 infections and COVID-19 hospitalisations and deaths in elderly long-term care facility residents, spain, weeks 532020 to 13 2021. Euro Surveill. 2021, 26, 2100452. [CrossRef] [PubMed]

13. Page, M.J.; McKenzie, J.E.; Bossuyt, P.M.; Boutron, I.; Hoffmann, T.C.; Mulrow, C.D.; Shamseer, L.; Tetzlaff, J.M.; Akl, E.A.; Brennan, S.E.; et al. The prisma 2020 statement: An updated guideline for reporting systematic reviews. BMJ 2021, 372, n71. [CrossRef]

14. Page, M.J.; McKenzie, J.E.; Bossuyt, P.M.; Boutron, I.; Hoffmann, T.C.; Mulrow, C.D.; Shamseer, L.; Tetzlaff, J.M.; Moher, D. Updating guidance for reporting systematic reviews: Development of the PRISMA 2020 statement. J. Clin. Epidemiol. 2021, 134, 103-112. [CrossRef]

15. Amit, S.; Regev-Yochay, G.; Afek, A.; Kreiss, Y.; Leshem, E. Early rate reductions of SARS-CoV-2 infection and COVID-19 in BNT162b2 vaccine recipients. Lancet 2021, 397, 875-877. [CrossRef]

16. Angel, Y.; Spitzer, A.; Henig, O.; Saiag, E.; Sprecher, E.; Padova, H.; Ben-Ami, R. Association between vaccination with BNT162b2 and incidence of symptomatic and asymptomatic SARS-CoV-2 infections among health care workers. JAMA 2021, 325, 2457-2465. [CrossRef]

17. Azamgarhi, T.; Hodgkinson, M.; Shah, A.; Skinner, J.A.; Hauptmannova, I.; Briggs, T.W.R.; Warren, S. BNT162b2 vaccine uptake and effectiveness in UK healthcare workers-A single centre cohort study. Nat. Commun. 2021, 12, 3698. [CrossRef]

18. Baum, U.; Poukka, E.; Palmu, A.A.; Salo, H.; Lehtonen, T.O.; Leino, T. Effectiveness of vaccination against SARS-CoV-2 infection and COVID-19 hospitalization among finnish elderly and chronically ill—An interim analysis of a nationwide cohort study. medRxiv 2021, in press. [CrossRef]

19. Benenson, S.; Oster, Y.; Cohen, M.J.; Nir-Paz, R. BNT162b2 mRNA COVID-19 Vaccine Effectiveness among Health Care Workers. N. Engl. J. Med. 2021, 384, 1775-1777. [CrossRef]

20. Lopez Bernal, J.; Andrews, N.; Gower, C.; Gallagher, E.; Simmons, R.; Thelwall, S.; Stowe, J.; Tessier, E.; Groves, N.; Dabrera, G.; et al. Effectiveness of COVID-19 Vaccines against the B.1.617.2 (Delta) Variant. N. Engl. J. Med. 2021, 385, 585-594. [CrossRef]

21. Bernal, J.L.; Andrews, N.; Gower, C.; Stowe, J.; Tessier, E.; Simmons, R.; Ramsay, M. Effectiveness of BNT162b2 mRNA vaccine and chadox1 adenovirus vector vaccine on mortality following COVID-19. medRxiv 2021, in press. [CrossRef]

22. Bianchi, F.P.; Germinario, C.A.; Migliore, G.; Vimercati, L.; Martinelli, A.; Lobifaro, A.; Tafuri, S.; Stefanizzi, P. BNT162b2 mRNA COVID-19 vaccine effectiveness in the prevention of SARS-CoV-2 infection: A preliminary report. J. Infect. Dis. 2021, 224, 431-434. [CrossRef]

23. Björk, J.; Inghammar, M.; Moghaddassi, M.; Rasmussen, M.; Malmqvist, U.; Kahn, F. Effectiveness of the BNT162b2 vaccine in preventing COVID-19 in the working age population-First results from a cohort study in southern sweden. medRxiv 2021, in press. [CrossRef]

24. Chodick, G.; Tene, L.; Patalon, T.; Gazit, S.; Ben Tov, A.; Cohen, D.; Muhsen, K. Assessment of effectiveness of 1 dose of BNT162b2 vaccine for SARS-CoV-2 infection 13 to 24 days after immunization. JAMA Netw. Open 2021, 4, e2115985. [CrossRef] [PubMed]

25. Chodick, G.; Tene, L.; Rotem, R.S.; Patalon, T.; Gazit, S.; Ben-Tov, A.; Weil, C.; Goldshtein, I.; Twig, G.; Cohen, D.; et al. The effectiveness of the two-dose BNT162b2 vaccine: Analysis of real-world data. Clin. Infect. Dis. 2021, in press. [CrossRef]

26. Chung, H.; He, S.; Nasreen, S.; Sundaram, M.E.; Buchan, S.A.; Wilson, S.E.; Chen, B.; Calzavara, A.; Fell, D.B.; Austin, P.C.; et al. Effectiveness of BNT162b2 and mRNA-1273 COVID-19 vaccines against symptomatic SARS-CoV-2 infection and severe COVID-19 outcomes in Ontario, Canada. medRxiv 2021, in press. [CrossRef] 
27. Corchado-Garcia, J.; Puyraimond-Zemmour, D.; Hughes, T.; Cristea-Platon, T.; Lenehan, P.; Pawlowski, C.; Bade, S.; O'Horo, J.C.; Gores, G.J.; Williams, A.W.; et al. Real-world effectiveness of Ad26.COV2.S adenoviral vector vaccine for COVID-19. medRxiv 2021, in press. [CrossRef]

28. Dagan, N.; Barda, N.; Kepten, E.; Miron, O.; Perchik, S.; Katz, M.A.; Hernán, M.A.; Lipsitch, M.; Reis, B.; Balicer, R.D. BNT162b2 mRNA COVID-19 vaccine in a nationwide mass vaccination setting. N. Engl. J. Med. 2021, 384, 1412-1423. [CrossRef]

29. Daniel, W.; Nivet, M.; Warner, J.; Podolsky, D.K. Early evidence of the effect of SARS-CoV-2 vaccine at one medical center. N. Engl. J. Med. 2021, 384, 1962-1963. [CrossRef]

30. Fabiani, M.; Ramigni, M.; Gobbetto, V.; Mateo-Urdiales, A.; Pezzotti, P.; Piovesan, C. Effectiveness of the comirnaty (BNT162b2, biontech/pfizer) vaccine in preventing SARS-CoV-2 infection among healthcare workers, Treviso Province, Veneto Region, Italy, 27 December 2020 To 24 March 2021. Euro Surveill. 2021, 26, 2100420. [CrossRef]

31. Flacco, M.; Soldato, G.; Martellucci, C.A.; Carota, R.; Di Luzio, R.; Caponetti, A.; Manzoli, L. Interim Estimates of COVID-19 Vaccine Effectiveness in a Mass Vaccination Setting: Data from an Italian Province. Vaccines 2021, 9, 628. [CrossRef]

32. Glampson, B.; Brittain, J.; Kaura, A.; Mulla, A.; Mercuri, L.; Brett, S.J.; Aylin, P.; Sandall, T.; Goodman, I.; Redhead, J.; et al. North west london COVID-19 vaccination programme: Real-world evidence for vaccine uptake and effectiveness. medRxiv 2021, in press. [CrossRef]

33. Haas, E.J.; Angulo, F.J.; McLaughlin, J.M.; Anis, E.; Singer, S.R.; Khan, F.; Brooks, N.; Smaja, M.; Mircus, G.; Pan, K.; et al. Impact and effectiveness of mRNA BNT162b2 vaccine against SARS-CoV-2 infections and COVID-19 cases, hospitalisations, and deaths following a nationwide vaccination campaign in Israel: An observational study using national surveillance data. Lancet 2021, 397, 1819-1829. [CrossRef]

34. Hall, V.J.; Foulkes, S.; Saei, A.; Andrews, N.; Oguti, B.; Charlett, A.; Wellington, E.; Stowe, J.; Gillson, N.; Atti, A.; et al. COVID-19 vaccine coverage in health-care workers in England and effectiveness of BNT162b2 mRNA vaccine against infection (SIREN): A prospective, multicentre, cohort study. Lancet 2021, 397, 1725-1735. [CrossRef]

35. Hitchings, M.; Ranzani, O.T.; Torres, M.S.S.; de Oliveira, S.B.; Almiron, M.; Said, R.; Borg, R.; Schulz, W.L.; de Oliveira, R.D.; da Silva, P.V.; et al. Effectiveness of coronavac among healthcare workers in the setting of high SARS-CoV-2 gamma variant transmission in manaus, Brazil: A test-negative case-control study. medRxiv 2021, in press. [CrossRef]

36. Jones, N.K.; Rivett, L.; Seaman, S.; Samworth, R.J.; Warne, B.; Workman, C.; Ferris, M.; Wright, J.; Quinnell, N.; Shaw, A.; et al. Single-dose BNT162b2 vaccine protects against asymptomatic SARS-CoV-2 infection. eLife 2021, 10, e68808. [CrossRef] [PubMed]

37. Lopez Bernal, J.; Andrews, N.; Gower, C.; Robertson, C.; Stowe, J.; Tessier, E.; Simmons, R.; Cottrell, S.; Roberts, R.; O'Doherty, M.; et al. Effectiveness of the pfizer-biontech and oxford-astrazeneca vaccines on COVID-19 related symptoms, hospital admissions, and mortality in older adults in england: Test negative case-control study. BMJ 2021, 373, n1088. [CrossRef]

38. Lumley, S.F.; Rodger, G.; Constantinides, B.; Sanderson, N.; Chau, K.K.; Street, T.L.; O'Donnell, D.; Howarth, A.; Hatch, S.B.; Marsden, B.D.; et al. An observational cohort study on the incidence of SARS-CoV-2 infection and b.1.1.7 variant infection in healthcare workers by antibody and vaccination status. Clin. Infect. Dis. 2021, ciab608. [CrossRef]

39. Moustsen-Helms, I.R.; Emborg, H.-D.; Nielsen, J.; Nielsen, K.F.; Krause, T.G.; Mølbak, K.; Møller, K.L.; Berthelsen, A.-S.N.; Valentiner-Branth, P. Vaccine effectiveness after 1st and 2nd dose of the BNT162b2 mRNA COVID-19 vaccine in long-term care facility residents and healthcare workers-A danish cohort study. medRxiv 2021, in press. [CrossRef]

40. Pawlowski, C.; Lenehan, P.; Puranik, A.; Agarwal, V.; Venkatakrishnan, A.; Niesen, M.J.; O'Horo, J.C.; Virk, A.; Swift, M.D.; Badley, A.D.; et al. FDA-authorized mRNA COVID-19 vaccines are effective per real-world evidence synthesized across a multi-state health system. Med 2021, 2, 979-992.E8. [CrossRef] [PubMed]

41. Pilishvili, T.; Fleming-Dutra, K.E.; Farrar, J.L.; Gierke, R.; Mohr, N.M.; Talan, D.A.; Krishnadasan, A.; Harland, K.K.; Smithline, H.A.; Hou, P.C.; et al. Interim estimates of vaccine effectiveness of pfizer-biontech and moderna COVID-19 vaccines among health care personnel-33 U.S. Sites, January-March 2021. MMWR Morb. Mortal. Weekl. Rep. 2021, 70, 753-758. [CrossRef]

42. Pritchard, E.; Matthews, P.C.; Stoesser, N.; Eyre, D.W.; Gethings, O.; Vihta, K.D.; Jones, J.; House, T.; VanSteenHouse, H.; Bell, I.; et al. Impact of vaccination on new SARS-CoV-2 infections in the united kingdom. Nat. Med. 2021, 27, 1370-1378. [CrossRef]

43. Ranzani, O.T.; Hitchings, M.; Dorion, M.; D'Agostini, T.L.; de Paula, R.C.; de Paula, O.F.P.; de Moura Villela, E.F.; Torres, M.S.S.; de Oliveira, S.B.; Schulz, W.; et al. Effectiveness of the coronavac vaccine in the elderly population during a p.1 variant-associated epidemic of COVID-19 in Brazil: A test-negative case-control study. medRxiv 2021, in press. [CrossRef]

44. Shrestha, N.K.; Nowacki, A.S.; Burke, P.C.; Terpeluk, P.; Gordon, S.M. Effectiveness of mRNA COVID-19 vaccines among employees in an american healthcare system. medRxiv 2021, in press. [CrossRef]

45. Shrotri, M.; Krutikov, M.; Palmer, T.; Giddings, R.; Azmi, B.; Subbarao, S.; Fuller, C.; Irwin-Singer, A.; Davies, D.; Tut, G.; et al. Vaccine effectiveness of the first dose of chadox $1 \mathrm{nCoV}-19$ and BNT162b2 against SARS-CoV-2 infection in residents of long-term care facilities (vivaldi study). Lancet 2021, 21, 1529-1538. [CrossRef]

46. Skowronski, D.M.; Setayeshgar, S.; Zou, M.; Prystajecky, N.; Tyson, J.R.; Galanis, E.; Naus, M.; Patrick, D.M.; Sbihi, H.; Adam, S.E.; et al. Single-dose mRNA vaccine effectiveness against SARS-CoV-2, including p.1 and b.1.1.7 variants: A test-negative design in adults 70 years and older in British Columbia, Canada. Clin. Infect. Dis. 2021, ciab616. [CrossRef]

47. Swift, M.D.; Breeher, L.E.; Tande, A.J.; Tommaso, C.P.; Hainy, C.M.; Chu, H.; Murad, M.H.; Berbari, E.F.; Virk, A. Effectiveness of mRNA COVID-19 vaccines against SARS-CoV-2 infection in a cohort of healthcare personnel. Clin. Infect. Dis. 2021, 73, e1376-e1379. [CrossRef] [PubMed] 
48. Thompson, M.G.; Burgess, J.L.; Naleway, A.L.; Tyner, H.; Yoon, S.K.; Meece, J.; Olsho, L.E.W.; Caban-Martinez, A.J.; Fowlkes, A.L.; Lutrick, K.; et al. Prevention and Attenuation of COVID-19 with the BNT162b2 and mRNA-1273 Vaccines. N. Engl. J. Med. 2021, 385, 320-329. [CrossRef] [PubMed]

49. Vahidy, F.S.; Pischel, L.; Tano, M.E.; Pan, A.P.; Boom, M.L.; Sostman, H.D.; Nasir, K.; Omer, S.B. Real world effectiveness of COVID-19 mRNA vaccines against hospitalizations and deaths in the united states. medRxiv 2021, in press. [CrossRef]

50. Vasileiou, E.; Simpson, C.R.; Shi, T.; Kerr, S.; Agrawal, U.; Akbari, A.; Bedston, S.; Beggs, J.; Bradley, D.; Chuter, A.; et al. Interim findings from first-dose mass COVID-19 vaccination roll-out and COVID-19 hospital admissions in scotland: A national prospective cohort study. Lancet 2021, 397, 1646-1657. [CrossRef]

51. Teo, S.P. Review of COVID-19 Vaccines and Their Evidence in Older Adults. Ann. Geriatr. Med. Res. 2021, 25, 4-9. [CrossRef]

52. Van Praet, J.T.; Vandecasteele, S.; De Roo, A.; De Vriese, A.S.; Reynders, M. Humoral and cellular immunogenicity of the BNT162b2 mRNA COVID-19 vaccine in nursing home residents. Clin. Infect. Dis. 2021, 73, 2145-2147. [CrossRef] [PubMed]

53. Brockman, M.A.; Mwimanzi, F.; Sang, Y.; Ng, K.; Agafitei, O.; Ennis, S.; Lapointe, H.; Young, L.; Umviligihozo, G.; Burns, L.; et al. Weak humoral immune reactivity among residents of long-term care facilities following one dose of the BNT162b2 mRNA COVID-19 vaccine. medRxiv 2021, in press. [CrossRef]

54. Canaday, D.H.; Carias, L.; Oyebanji, O.A.; Keresztesy, D.; Wilk, D.; Payne, M.; Aung, H.; St Denis, K.; Lam, E.C.; Rowley, C.F.; et al. Reduced BNT162b2 mRNA vaccine response in SARS-CoV-2-naive nursing home residents. medRxiv 2021, in press. [CrossRef]

55. Müller-Sedgwick, U. Optimal dosing interval for COVID-19 vaccines: We need clinical trial evidence and to consider past infection. BMJ 2021, 372, n522. [CrossRef]

56. Iacobucci, G.; Mahase, E. COVID-19 vaccination: What's the evidence for extending the dosing interval? BMJ 2021, $372, \mathrm{n} 18$. [CrossRef]

57. Bernal, J.L.; Andrews, N.; Gower, C.; Stowe, J.; Robertson, C.; Tessier, E.; Simmons, R.; Cottrell, S.; Roberts, R.; O'Doherty, M.; et al. Early effectiveness of COVID-19 vaccination with BNT162b2 mRNA vaccine and chadox1 adenovirus vector vaccine on symptomatic disease, hospitalisations and mortality in older adults in England. medRxiv 2021, in press. [CrossRef] 